\title{
ON THE OPTIMAL CONTROL OF IMPLICIT SYSTEMS
}

\author{
PHILIPPE PETIT
}

ABSTRACT. In this paper we consider the following problem, known as implicit Lagrange problem: find the trajectory $x$ argument of

$$
\min \int_{0}^{1} L(x, \dot{x}) d t
$$

where the constraints are defined by an implicit differential equation

$$
F(x, \dot{x})=0
$$

with $\operatorname{dim} F=n-q<\operatorname{dim} x=n$. We define the geometric framework of a $q-\pi$-submanifold in the tangent bundle of a surrounding manifold $X$, which is an extension of the $\pi$-submanifold geometric framework defined by Rabier and Rheinboldt for control systems. With this geometric framework, we define a class of well-posed implicit differential equations for which we obtain locally a controlled vector field on a submanifold $W$ of the surrounding manifold $X$ by means of a reduction procedure. We then show that the implicit Lagrange problem leads locally to an explicit optimal control problem on the submanifold $W$, for which the Pontryagin maximum principle is naturally apply.

\section{INTRODUCTION}

We consider for the state $x$ of $\mathbb{R}^{n}$ the implicit differential equation

$$
F(x, \dot{x})=0 .
$$

In this equation the control $u$ does not appear explicitly, but only because there are less equations than unknowns, namely $F: \mathbb{R}^{n} \times \mathbb{R}^{n} \rightarrow \mathbb{R}^{n-q}$, where $q<n$ (see [6].) Here, the control variable $u$ belongs to $\mathbb{R}^{q}$. The cost function is the Lagrangian $L(x, \dot{x})$ of $T \mathbb{R}^{n}$. A process is a trajectory $x(\cdot)$ belonging to $C^{1}\left([0,1], \mathbb{R}^{n}\right)$ the set of continously differentiable functions (resp. $K C^{1}\left([0,1], \mathbb{R}^{n}\right)$ the set of continuous and piecewise differentiable functions, $A C\left([0,1], \mathbb{R}^{n}\right)$ the set of absolutely continuous functions, see the footnotes of the subsection 5.2.) A trajectory $x(\cdot)$ is admissible if $x(0)=a$, $x(1)=b$ and

$$
F(x(t), \dot{x}(t))=0, \forall t \in[0,1] \text { (resp. a.e. on }[0,1]) .
$$

For any admissible trajectory $x(\cdot)$ the cost is

$$
J(x(\cdot))=\int_{0}^{1} L(x(t), \dot{x}(t)) d t .
$$

Institut de mathématiques, UMR C9994 du CNRS, Université Paris 7, UFR de mathématiques, CASE 7012, 2, place Jussieu F-75251 Paris Cedex 05, France. E-mail: petit@labomath.univ-orleans.fr.

Received by the journal April 29, 1997. Revised September 1997. Accepted for publication January 5, 1998.

(C) Société de Mathématiques Appliquées et Industrielles. Typeset by LATEX. 
An admissible trajectory $\bar{x}(\cdot)$ belonging to $C^{1}\left([0,1], \mathbb{R}^{n}\right.$ ) (resp. $\left.K C^{1}\left([0,1], \mathbb{R}^{n}\right), A C\left([0,1], \mathbb{R}^{n}\right)\right)$ is a weak minimum (resp. strong minimum) of $J$ if

$$
J(\bar{x}(\cdot)) \leq J(x(\cdot))
$$

for any admissible trajectory $x(\cdot)$ belonging to $C^{1}\left([0,1], \mathbb{R}^{n}\right.$ ) (resp. $\left.K C^{1}\left([0,1], \mathbb{R}^{n}\right), A C\left([0,1], \mathbb{R}^{n}\right)\right)$. An admissible trajectory $\bar{x}(\cdot)$ belonging to $C^{1}\left([0,1], \mathbb{R}^{n}\right)\left(\operatorname{resp.} K C^{1}\left([0,1], \mathbb{R}^{n}\right), A C\left([0,1], \mathbb{R}^{n}\right)\right)$ is a weak local minimum (resp. strong local minimum) of $J$ if there exist an $\varepsilon>0$ such that for any trajectory $x(\cdot)$ belonging to $C^{1}\left([0,1], \mathbb{R}^{n}\right)$ (resp. $K C^{1}\left([0,1], \mathbb{R}^{n}\right)$, $\left.A C\left([0,1], \mathbb{R}^{n}\right)\right)$ such that $\|x(\cdot)-\bar{x}(\cdot)\|_{1}<\varepsilon\left(\operatorname{resp} .\|x(\cdot)-\bar{x}(\cdot)\|_{0}<\varepsilon\right)$ where

$$
\begin{gathered}
\|x(\cdot)\|_{1}=\max _{t \in[0,1]} \max \{|x(t)|,|\dot{x}(t)|\} \\
\left(\text { resp. }\|x(\cdot)\|_{0}=\max _{t \in[0,1]}|x(t)|\right)
\end{gathered}
$$

then

$$
J(\bar{x}(\cdot)) \leq J(x(\cdot))
$$

REMARK 1.1. a) An admissible trajectory $x(\cdot)$ belonging to $C^{1}\left([0,1], \mathbb{R}^{n}\right)$ which is a strong (local) minimum is also a weak (local) minimum, meanwhile a trajectory $x(\cdot)$ belonging to $C^{1}\left([0,1], \mathbb{R}^{n}\right)$ can be a weak (local) minimum without to be a strong (local) minimum.

b) The necessary conditions for the weak local minimum are also necessary conditions for the strong local minimum, and the sufficiency conditions for the strong local minimum are also the sufficiency conditions for the weak local minimum.

We will subsequently turn our attention to the geometry of the implicit differential equation (1.1). More precisely, we will extend the definitions of $\pi$-submanifold, reducible and completely reducible $\pi$-submanifold in [16] to our situation (see also [15].) Let us consider the manifold $X=\mathbb{R}^{n}$ and its tangent bundle $T X=T \mathbb{R}^{n}=\mathbb{R}^{n} \times \mathbb{R}^{n}$. Let us assume that the subset $M=F^{-1}(0)$ is a submanifold of $T X$ (it is the case when $F$ is a submersion). A trajectory $x(\cdot)$ is admissible if $(x(t), \dot{x}(t))$ belongs to $M$ for any $t \in[0,1]$. The implicit Lagrange problem ${ }^{1}$ is

$$
\mathcal{P}_{0} \quad \min _{(x(\cdot), \dot{x}(\cdot)) \in M} \int_{0}^{1} L(x(t), \dot{x}(t)) d t .
$$

If $x(\cdot)$ is an admissible trajectory then $x(t)$ has to belong to the set $W=$ $\pi(M)$ for any $t \in[0,1]$. Let us assume that $W$ is a submanifold of $X$, then $\dot{x}(t)$ has to belong to the subspace $T_{x(t)} W$ of $T_{x(t)} X$ for any $t$ in $[0,1]$ and thus $(x(t), \dot{x}(t))$ belongs to the set $M_{1}=T W \cap M$ for any $t$ in $[0,1]$. In other

\footnotetext{
${ }^{1}$ When $q=n$, the constraint $F(x, \dot{x})=0$ being absent, this is the simple problem of the calculus of variations (see $[3,4,5,10,11]$ )

$$
\min _{\substack{x(0)=a \\ x(1)=b}} \int_{0}^{1} L(x(t), \dot{x}(t)) d t .
$$
}


words, any admissible trajectory for the implicit Lagrange problem $\left(\mathcal{P}_{0}\right)$ is an admissible trajectory for the following implicit Lagrange problem

$$
\mathcal{P}_{1} \quad \min _{(x(\cdot), \dot{x}(\cdot)) \in M_{1}} \int_{0}^{1} L(x(t), \dot{x}(t)) d t .
$$

Thus, if $\bar{x}(\cdot)$ is a solution of $\mathcal{P}_{0}$ then it is a solution of $\mathcal{P}_{1}$ and conversely. Moreover, the startpoint $a$ and the endpoint $b$ have to belong to $W$. This replacement of the submanifold $M$ of $T X$ by the submanifold $M_{1}$ of $T X$ is the reduction procedure and $M_{1}$ is called the reduction of $M$. Let us assume that we are able to do with $M_{1}$, what we have done with $M$, then we construct a submanifold $W_{1}=\pi\left(M_{1}\right)$ of $X$ and a submanifold $M_{2}$ of $T X$. If $x(\cdot)$ is an admissible trajectory for the problem $\mathcal{P}_{1}$ then it is an admissible trajectory for the problem

$\mathcal{P}_{2}$

$$
\min _{\substack{x(\cdot), \dot{x}(\cdot)) \in M_{2} \\ x(0)=a \\ x(1)=b}} \int_{0}^{1} L(x(t), \dot{x}(t)) d t .
$$

Let us assume that we construct by induction a sequence of implicit Lagrange problem

$\mathcal{P}_{k}$

$$
\begin{aligned}
& \min _{(x(\cdot), \dot{x}(\cdot)) \in M_{k}} \\
& x(0)=a \\
& x(1)=b
\end{aligned}
$$

such that $W_{k}=\pi\left(M_{k}\right)$ is a submanifold of $X$ and $M_{k+1}=M_{k} \cap T W_{k}$ is a submanifold of $T X$, then any admissible trajectory $x(\cdot)$ of $\mathcal{P}_{k}$ is an admissible trajectory of $\mathcal{P}_{k+1}$. Therefore any admissible trajectory of $\mathcal{P}_{0}$ is an admissible trajectory of $\mathcal{P}_{k}$ for any $k$. Thus any admissible trajectory of $\mathcal{P}_{0}$ is an admissible trajectory of the following implicit Lagrange problem

$$
\mathcal{P}_{c} \quad \min _{(x(\cdot), \dot{x}(\cdot)) \in C(M)} \int_{0}^{1} L(x(t), \dot{x}(t)) d t
$$

with $C(M)=\cap_{k>0} M_{k}$. Clearly, the strong (resp. weak) minimum of $\mathcal{P}_{0}$ are the strong (resp. weak) minimum of $\mathcal{P}_{c}$ and the points $a$ et $b$ have to belong to $\pi(C(M))$. Furthermore, if the sequence $\left\{M_{k}\right\}_{k>0}$ is stationary then $C(M)$ is a submanifold of $T X$ and the smallest integer $\alpha$ such that $M_{k}=M_{\alpha}, \forall i \geq$ $\alpha$ will be called the index. Then we can wonder if $\mathcal{P}_{c}$ is equivalent to an explicit control problem (see the subsection 5.2 of the Appendix). This will be the case for the class of well-posed implicit differential equations.

\section{DEFINITIONS AND MAIN RESUlTS}

Using the geometric framework of $q$ - $\pi$-submanifold of the section 3 , we are able to define a well-posed implicit differential equation. 
Definition 2.1. An implicit differential equation (1.1) is well-posed if the set

$$
M=F^{-1}(0)
$$

is a completely reducible $q-\pi$-submanifold of $T \mathbb{R}^{n}$ such that the core $C(M)$ is not empty.

Definition 2.2. The index of a well-posed implicit differential equation (1.1) is the maximum over the index of the non-empty connected component of the core $C(M)$.

Now, we consider only well-posed implicit differential equations. As in the introduction, for each $q-\pi$-submanifold $M_{k}$ of $T \mathbb{R}^{n}$ of the chain of reduction we consider the implicit Lagrange problem

$\mathcal{P}_{k}$

$$
\begin{aligned}
& \min _{(x(\cdot), \dot{x}(\cdot)) \in M_{k}} \int_{0}^{1} L(x(t), \dot{x}(t)) d t \\
& x(0)=a \\
& x(1)=b
\end{aligned}
$$

and for $C(M)$ the core of $M$ we consider the implicit Lagrange problem

$$
\begin{aligned}
& \mathcal{P}_{c} \quad \min _{(x(\cdot), \dot{x}(\cdot)) \in C(M)} \int_{0}^{1} L(x(t), \dot{x}(t)) d t . \\
& x(0)=a \\
& x(1)=b
\end{aligned}
$$

The sequence $\left\{\mathcal{P}_{k}\right\}_{k \geq 0}$ is called the chain of reduced implicit Lagrange problems of the well-posed implicit differential equation (1.1) and $\mathcal{P}_{c}$ is called the central implicit Lagrange problem. Evidently, the points $a$ and $b$ have to belong to $\pi\left(M_{k}\right)$ for any $k$ and thus to belong to $\pi(C(M))$.

Definition 2.3. Any point $x$ of $\mathbb{R}^{n}$ is consistent with a well-posed implicit differential equation $F$ if it belongs to the projection of the core $C(M)$.

According to the definitions we can formulate the following theorems (proofs are given in the subsection 5.1 of the Appendix).

Theorem 2.4. Let $F$ be a well-posed implicit differential equation $(q<n)$ of $T \mathbb{R}^{n},\left\{P_{k}\right\}_{k>0}$ its chain of reduced implicit Lagrange problems and $\mathcal{P}_{c}$ its central implicit Lagange's problem. Then, any admissible trajectory $x(\cdot)$ of $\mathcal{P}_{0}$ is an admissible trajectory of $\mathcal{P}_{k}$ for any $k$. In particular, any admissible trajectory $x(\cdot)$ of $\mathcal{P}_{0}$ is an admissible trajectory of $\mathcal{P}_{c}$ and any strong (resp. weak) minimum $\bar{x}(\cdot)$ of $\mathcal{P}_{c}$ is a strong (resp. weak) minimum of $\mathcal{P}_{0}$.

Theorem 2.4 shows that the strong (resp. weak) minimum are living in the core $C(M)$. According to the theorem 3.31, the $q-\pi$-submanifold $C(M)$ is locally the image of a controlled vector field. Thus, we are able to show that locally the trajectories of $C(M)$ are in bijection with the trajectories of the controlled vector field $\chi$.

Theorem 2.5. (Local equivalence) Let $F$ be a well-posed implicit differential equation, $C(M)$ its core, $\left(x_{0}, p_{0}\right)$ a point belonging to $C(M)$ and $W=\pi(V)$ a local projection of $C(M)$ at $\left(x_{0}, p_{0}\right), O$ an open set of $\mathbb{R}^{q}$, $x$ a controlled vector field given by the theorem 3.31. If $x(\cdot)$ is a local trajectory of $C(M)$ such that $(x(t), \dot{x}(t))$ belongs to $V$ for any $t$, then there ESAIM: COCV, MARCH 1998, VOL. 3, 49-81 
exists an unique continuous (resp. piecewise continuous) control $u(\cdot)$ taking its value in $O$ such that $(x(t), \dot{x}(t))=\chi(x(t), u(t))^{2}$ for any $t$. Conversely, for any initial condition $x_{0}$ belonging to $W$ and for any continuous (resp. piecewise continuous) control $u(\cdot)$ taking its value in $O$ there exists a unique local trajectory of $C(M)$ such that $(x(t), \dot{x}(\cdot))$ belongs to $V$ for any $t$.

Then, on the one hand, we have shown that the strong (resp. weak) minimum of the implicit Lagrange problem $\mathcal{P}_{0}$ are the strong (resp. weak) minimum of the central implicit Lagrange problem $\mathcal{P}_{c}$ (theorem 2.4), and on the other hand that (locally) the admissible trajectories of $\mathcal{P}_{c}$ are in bijection with the admissible trajectories of the controlled vector field $\chi$ (theorem 2.5). Now, let us consider a strong minimum $\bar{x}(\cdot)$ of the central implicit Lagrange problem $\mathcal{P}_{c}$. Let $\tau$ be a point in the set $T$ and $W=\pi(V)$ a local projection of $C(M)$ at $(\bar{x}(\tau), \dot{\bar{x}}(\tau)), O$ an open set of $\mathbb{R}^{q}, \chi$ a controlled vector field given by the theorem 3.31. There exists $\varepsilon>0$ such that for any point $t$ belonging to the interval $I_{\varepsilon}=[\tau-\varepsilon, \tau+\varepsilon]$ then $(\bar{x}(t), \dot{\bar{x}}(t))$ belongs to $V$. We naturally consider the following local implicit Lagrange problem

$$
\mathcal{P}_{c, \varepsilon} \min _{(x(\cdot), \dot{x}(\cdot)) \in V} \int_{\tau-\varepsilon}^{\tau+\varepsilon} L(x(t), \dot{x}(t)) d t .
$$

Theorem 2.6. (Local optimality) Let $F$ be a well-posed implicit differential equation. If $\bar{x}(\cdot)$ is a strong minimum of the central implicit Lagrange problem $\mathcal{P}_{c}$ then for any $\tau$ belonging to $T$ there exists $\varepsilon>0$ such that the trajectory $\dot{\bar{x}}_{\mid I_{\varepsilon}}(\cdot)$ is a strong minimum of the implicit Lagrange problem $\mathcal{P}_{c, \varepsilon}$

Let us also consider the following local explicit optimal control problem

$$
\begin{gathered}
\min _{e, \varepsilon} \\
(x(\cdot), \dot{x}(\cdot))=\chi(x(\cdot), u(\cdot)) \\
u(\cdot) \in O \\
x(\tau-\varepsilon)=\bar{x}(\tau-\varepsilon) \\
x(\tau+\varepsilon)=\bar{x}(\tau+\varepsilon)
\end{gathered}
$$

THEOREM 2.7. $\bar{x}(\cdot)$ is strong (local) minimum of the implicit Lagrange problem $\mathcal{P}_{c, \varepsilon}$ if, and only if, the corresponding admissible process $(\bar{x}(\cdot), \bar{u}(\cdot))$ is a strong (local) minimum for the explicit control problem $\mathcal{P}_{e, \varepsilon}$.

This leads to consider the following local implicit Lagrange problem. Let $W=\pi(V)$ be a local projection such that there exists an open set $O$ in $\mathbb{R}^{q}$ and a controlled vector field $\chi: W \times O \rightarrow T W$ given by the theorem 3.31

$$
\begin{aligned}
& \min _{(x(\cdot), \dot{x}(\cdot)) \in V} \int_{0}^{1} L(x(t), \dot{x}(t)) d t \\
& x(0)=a \\
& x(1)=b
\end{aligned}
$$

where the points $a$ and $b$ belong to $W$. The admissible trajectories (resp. strong minimum) of $\mathcal{P}_{V}$ are in bijection with the admissible processes (resp.

\footnotetext{
${ }^{2}$ In a local coordinate system $x$ of $W, \chi(x, u)$ takes the form $(x, f(x, u))$.
} 
strong minimum) of the explicit optimal control problem

$\mathcal{P}_{e}$

$$
\begin{aligned}
& \min _{(x(\cdot), \dot{x}(\cdot))=\chi(x(\cdot), u(\cdot))} \int_{0}^{1} L(\chi(x(t), u(t)) d t . \\
& u(\cdot) \in O \\
& x(0)=a \\
& x(1)=b
\end{aligned}
$$

Finally, we choose a local coordinate system $x=\left(x^{1}, \cdots, x^{r_{\alpha}}\right)$ of $W$ and then apply the Maximum Principle to the problem $\mathcal{P}_{e}$ with the pseudoHamiltonian

$$
\begin{gathered}
H^{\psi_{0}}: T^{\star} W \times O \rightarrow \mathbb{R} \\
(x, \psi, u) \mapsto \sum_{i=1}^{r_{\alpha}} \psi_{i} f^{i}(x, u)+\psi_{0} L(x, f(x, u))
\end{gathered}
$$

and the controlled vector field

$$
\begin{aligned}
& \vec{H}^{\psi_{0}}: T^{\star} W \times O \rightarrow T\left(T^{\star} W\right) \\
& (x, \psi, u) \mapsto \vec{H}^{\psi_{0}}(x, \psi, u)=\sum_{i=1}^{r_{\alpha}} \frac{\partial H^{\psi_{0}}}{\partial \psi_{i}}(x, \psi, u) \frac{\partial}{\partial x^{i}}-\sum_{i=1}^{r_{\alpha}} \frac{\partial H^{\psi_{0}}}{\partial x^{i}}(x, \psi, u) \frac{\partial}{\partial \psi_{i}}
\end{aligned}
$$

where $\psi_{0}=0,1$.

REMARK 2.8. Obviously, the necessary conditions of optimality are invariant by bundle isomorphism $h$. Let us consider a bundle isomorphism $h$

$$
\tilde{x}=\tilde{X}(x), \tilde{u}=\tilde{U}(x, u)
$$

with inverse

$$
x=X(\tilde{x}), u=U(\tilde{x}, \tilde{u}) .
$$

In the new coordinates $(\tilde{x}, \tilde{u})$ the controlled vector field is

$$
\tilde{f}(\tilde{x}, \tilde{u})=\frac{\partial \tilde{X}}{\partial x}(X(\tilde{x})) f(X(\tilde{x}), U(\tilde{x}, \tilde{u})),
$$

the Lagrangian $\tilde{L}(\tilde{x}, \tilde{u})=L(X(\tilde{x}), U(\tilde{x}, \tilde{u}))$ and the pseudo-Hamiltonian

$$
\left.\tilde{H}(\tilde{x}, \tilde{\psi}, \tilde{u})=\sum_{i=1}^{r_{\alpha}} \tilde{\psi}_{i} \tilde{f}^{i}(\tilde{x}, \tilde{u})-\tilde{\psi}_{0} \tilde{L}(\tilde{x}, \tilde{u})\right) .
$$

The extremals $(\overline{\tilde{x}}(\cdot), \overline{\tilde{\psi}}(\cdot))$ are the projection of a triplet $(\overline{\tilde{x}}(\cdot), \overline{\tilde{\psi}}(\cdot), \overline{\tilde{u}}(\cdot))$ such that

$(\tilde{a}):(\overline{\tilde{x}}(\cdot), \overline{\tilde{\psi}}(\cdot), \overline{\tilde{u}}(\cdot))$ is a trajectory of the controlled vector field $\left(\tilde{\psi}_{0}=\right.$ $0,1)$

$$
\begin{gathered}
\overrightarrow{\hat{H}^{\psi_{0}}}: T^{\star} \tilde{W} \times \tilde{O} \rightarrow T\left(T^{\star} \tilde{W}\right) \\
(\tilde{x}, \tilde{\psi}, u) \mapsto \overrightarrow{\tilde{H}}^{\tilde{\psi}_{0}}(\tilde{x}, \tilde{\psi}, \tilde{u})=\sum_{i=1}^{r_{\alpha}} \frac{\partial \tilde{H}^{\tilde{\psi}_{0}}}{\partial \tilde{\psi}_{i}}(\tilde{x}, \tilde{\psi}, \tilde{u}) \frac{\partial}{\partial \tilde{x}^{i}}-\sum_{i=1}^{n} \frac{\partial \tilde{H}^{\tilde{\psi}_{0}}}{\partial \tilde{x}^{i}}(\tilde{x}, \tilde{\psi}, \tilde{u}) \frac{\partial}{\partial \tilde{\psi}_{i}}
\end{gathered}
$$

$(\tilde{b})$ : for any $t$ belonging to $[0,1]$ (resp. a.e. on $[0,1]$ )

$$
\tilde{H}^{\tilde{\psi}_{0}}(\overline{\tilde{x}}(t), \overline{\tilde{u}}(t), \overline{\tilde{\psi}}(t))=\max _{\tilde{u} \in \tilde{O}} \tilde{H}^{\tilde{\psi}_{0}}(\overline{\tilde{x}}(t), u, \overline{\tilde{\psi}}(t)) .
$$


Clearly, the extremals $(\overline{\tilde{x}}(\cdot), \overline{\tilde{\psi}}(\cdot))$ are in bijection with the extremals $(\bar{x}(\cdot), \bar{\psi}(\cdot))$ via the relationship

$$
(\overline{\tilde{x}}(\cdot), \overline{\tilde{\psi}}(\cdot))=\left(\tilde{X}\left(\bar{x}(\cdot), \frac{{ }^{t} \partial X}{\partial \tilde{x}}(\tilde{X}(\bar{x}(\cdot))) \bar{\psi}(\cdot)\right) .\right.
$$

For any triplet $(\bar{x}(\cdot), \bar{\psi}(\cdot), \bar{u}(\cdot))$ such that $(a)$ and $(b)$ are satisfied, the triplet

$$
(\overline{\tilde{x}}(\cdot), \overline{\tilde{\psi}}(\cdot), \overline{\tilde{u}}(\cdot))=\left(\tilde{X}(\bar{x}(\cdot)), \frac{{ }^{t} \partial X}{\partial \tilde{x}}(\tilde{X}(\bar{x}(\cdot))) \bar{\psi}(\cdot), \tilde{U}(\bar{x}(\cdot), \bar{u}(\cdot))\right)
$$

satisfies $(\tilde{a})$ and $(\tilde{b})$.

EXAMPLE 2.9. The controlled rigid pendulum. A mass $m$ is attached at the extremity of a rigid massless wire of length $l$ and fixed at the origin. $\tau$ is the tension of the wire, $g$ the gravity constant and the control $u=\left(u_{1}, u_{2}\right)$ acts on the mass. The equations of the system are

$$
\begin{aligned}
m \ddot{x}_{1} & =-\frac{\tau}{l} x_{1}+u_{1} \\
m \ddot{x}_{2} & =-\frac{\tau}{l} x_{2}+m g+u_{2} . \\
0 & =x_{1}^{2}+x_{2}^{2}-l^{2}
\end{aligned}
$$

In order to return to an implicit differential equation and to use the reduction procedure we consider the following mapping $F_{0}: T \mathbb{R}^{7} \rightarrow \mathbb{R}^{5}$

$$
F_{0}(x, p)=\left(\begin{array}{c}
p_{1}-x_{3} \\
p_{2}-x_{4} \\
p_{3}+x_{1} p_{7}-p_{5} \\
p_{4}+x_{2} p_{7}-p_{6}-g \\
x_{1}^{2}+x_{2}^{2}-l^{2}
\end{array}\right), \quad p=\dot{x}
$$

where $\dot{x}_{5}=u_{1} / m, \dot{x}_{6}=u_{2} / m$ and $\dot{x}_{7}=\tau / m l$ and the submanifold, of $T \mathbb{R}^{7}$, $M_{0}=F_{0}^{-1}(0)$. $M_{0}$ has dimension 9 . The equation of the set $W_{0}=\pi\left(M_{0}\right)$ is

$$
x_{1}^{2}+x_{2}^{2}-l^{2}=0
$$

it is a submanifold of $\mathbb{R}^{7}$ of dimension $6 . T W_{0}=G_{0}^{-1}(0)$ where $G_{0}$ is the mapping

$$
G_{0}(x, p)=\left(\begin{array}{c}
x_{1}^{2}+x_{2}^{2}-l^{2} \\
p_{1} x_{1}+p_{2} x_{2}
\end{array}\right) .
$$

Thus the reduction of $M_{0}$ is $M_{1}=T W_{0} \cap M_{0}=F_{1}^{-1}(0)$, where $F_{1}$ is the mapping

$$
F_{1}(x, p)=\left(\begin{array}{c}
p_{1}-x_{3} \\
p_{2}-x_{4} \\
p_{3}+x_{1} p_{7}-p_{5} \\
p_{4}+x_{2} p_{7}-p_{6}-g \\
x_{1}^{2}+x_{2}^{2}-l^{2} \\
x_{1} x_{3}+x_{2} x_{4}
\end{array}\right)
$$

it is a submanifold of dimension 8 . The equations of the set $W_{1}=\pi\left(M_{1}\right)$ are

$$
\begin{aligned}
x_{1}^{2}+x_{2}^{2}-l^{2}= & 0 \\
x_{1} x_{3}+x_{2} x_{4}= & 0 ; \\
& \text { ESAIM: CoCv, March 1998, Vol. } 3,49-81
\end{aligned}
$$


and it is a submanifold of $\mathbb{R}^{7}$ of dimension 5. $T W_{1}=G_{1}^{-1}(0)$, where $G_{1}$ is the mapping

$$
G_{1}(x, p)=\left(\begin{array}{c}
x_{1}^{2}+x_{2}^{2}-l^{2} \\
x_{1} x_{3}+x_{2} x_{4} \\
p_{1} x_{1}+p_{2} x_{2} \\
p_{1} x_{3}+x_{1} p_{3}+p_{2} x_{4}+x_{2} p_{4}
\end{array}\right)
$$

Thus $M_{2}=T W_{1} \cap M_{1}=F_{2}^{-1}(0)$, where $F_{2}$ is the mapping

$$
F_{2}(x, p)=\left(\begin{array}{c}
p_{1}-x_{3} \\
p_{2}-x_{4} \\
p_{3}+x_{1} p_{7}-p_{5} \\
p_{4}+x_{2} p_{7}-p_{6}-g \\
x_{1}^{2}+x_{2}^{2}-l^{2} \\
x_{1} x_{3}+x_{2} x_{4} \\
x_{3}^{2}+x_{4}^{2}+x_{1} p_{5}+x_{2} p_{6}+x_{2} g-l^{2} p_{7}
\end{array}\right),
$$

is a submanifold of dimension 7 . Finally the set $W_{2}=\pi\left(M_{2}\right)$ is in fact $W_{1}$ and thus $M_{3}=T W_{2} \cap M_{2}=T W_{1} \cap M_{2}=M_{2}$ (since $M_{2} \subset T W_{1}$ ), then $C\left(M_{0}\right)$ is the submanifold $M_{2}$ and $W=\pi\left(C\left(M_{0}\right)\right)$ is the submanifold $W_{2}$. Moreover $C(M)=\chi\left(W \times \mathbb{R}^{2}\right)$ where $\chi(x, v)=(x, f(x, v))$ is the vector field of the state $x \in W$ depending on $v=\left(v_{1}, v_{2}\right) \in \mathbb{R}^{2}$ such that

$$
f(x, v)=\left(\begin{array}{c}
x_{3} \\
x_{4} \\
-\frac{x_{1}}{l_{2}^{2}}\left(x_{3}^{2}+x_{4}^{2}\right)+\frac{x_{2}}{l^{2}}\left(x_{2} v_{1}-x_{1}\left(v_{2}+g\right)\right) \\
-\frac{x_{2}}{l^{2}}\left(x_{3}^{2}+x_{4}^{2}\right)-\frac{x_{1}}{l^{2}}\left(x_{2} v_{1}-x_{1}\left(v_{2}+g\right)\right) \\
v_{1} \\
v_{2} \\
\frac{1}{l^{2}}\left(x_{3}^{2}+x_{4}^{2}+x_{1} v_{1}+x_{2}\left(v_{2}+g\right)\right)
\end{array}\right)
$$

and $v_{1}=u_{1} / m$ et $v_{2}=u_{2} / m$. On the other hand, from the relation $x_{1}=$ $l \sin \theta$ and $x_{2}=l \cos \theta(\theta \in]-\pi, \pi[)$ we obtain

$$
\left\{\begin{array} { l } 
{ x _ { 3 } = l \dot { \theta } \operatorname { c o s } \theta } \\
{ x _ { 4 } = - l \dot { \theta } \operatorname { s i n } \theta }
\end{array} \left\{\begin{array}{l}
\dot{x}_{3}=-l \dot{\theta}^{2} \sin \theta+l \ddot{\theta} \cos \theta \\
\dot{x}_{4}=-l \dot{\theta}^{2} \cos \theta-l \ddot{\theta} \sin \theta \\
\dot{x}_{7}=\dot{\theta}^{2}+v_{1} \frac{\sin \theta}{l}+v_{2} \frac{\cos \theta}{l}
\end{array}\right.\right.
$$

Therefore, $\theta$ satisfy the following second order implicit differential equation

$$
\ddot{l} \ddot{\theta}=v_{1} \cos \theta-\left(v_{2}+g\right) \sin \theta
$$

Thus, we take for $W$ the parameterization

$$
x=X(z)=\left(l \sin \theta, l \cos \theta, l \vartheta \cos \theta,-l \vartheta \sin \theta, y_{5}, y_{6}, y_{7}\right),
$$

where $\left.z=\left(\theta, \vartheta, y_{5}, y_{6}, y_{7}\right) \in\right]-\pi, \pi\left[\times \mathbb{R}^{4}\right.$, the controlled vector field

$$
\begin{aligned}
\chi(z, v)=(z, g(z, v))= & \vartheta \frac{\partial}{\partial \theta}+\left(v_{1} \frac{\cos \theta}{l}-\left(v_{2}+g\right) \frac{\sin \theta}{l}\right) \frac{\partial}{\partial \vartheta}+v_{1} \frac{\partial}{\partial y_{5}} \\
& +v_{2} \frac{\partial}{\partial y_{6}}+\left(\vartheta^{2}+v_{1} \frac{\sin \theta}{l}+\left(v_{2}+g\right) \frac{\cos \theta}{l}\right) \frac{\partial}{\partial y_{7}}
\end{aligned}
$$


and the Lagrangian $\tilde{L}(z, v)=L\left(X(z), \frac{\partial X}{\partial z}(z) g(z, v)\right)$. Then the problem $\mathcal{P}_{c}$ is equivalent to the explicit optimal control problem

$$
\begin{gathered}
\min _{(z(t), \dot{z}(t))=\chi(z(t), v(t))} \\
v(t) \in \mathbb{R}^{2} \\
z(0) \in\{a\} \times \mathbb{R}^{3} \\
z(1) \in\{b\} \times \mathbb{R}^{3}
\end{gathered}
$$

for which we obtain the necessary conditions of optimality with the pseudoHamiltonian

$$
\begin{aligned}
H^{\psi_{0}}(z, \psi, v)= & \psi_{1} \vartheta+\psi_{2}\left(v_{1} \frac{\cos \theta}{l}-\left(v_{2}+g\right) \frac{\sin \theta}{l}\right)+\psi_{3} v_{1}+\psi_{4} v_{2} \\
& +\psi_{5}\left(\vartheta^{2}+v_{1} \frac{\sin \theta}{l}+\left(v_{2}+g\right) \frac{\cos \theta}{l}\right)-\psi_{0} \tilde{L}(z, v)
\end{aligned}
$$

REMARK 2.10. For this system, the kinetic energy is $T(\theta, \dot{\theta})=\frac{1}{2} m l^{2} \dot{\theta}^{2}$, the potential energy is $V(\theta)=-m g l \cos \theta$ and the Lagrangian is

$$
\mathrm{L}=T-V=\frac{1}{2} m l^{2} \dot{\theta}^{2}+m g l \cos \theta .
$$

The virtual work of the control $u$ is $\delta W_{u}=Q \delta \theta=\left(u_{1} l \cos \theta-u_{2} l \sin \theta\right) \delta \theta$ and for the tension it is zero. The Lagrange equation

$$
\frac{d}{d t} \frac{\partial \mathrm{L}}{\partial \dot{\theta}}-\frac{\partial \mathrm{L}}{\partial \theta}=Q
$$

gives the second order differential equation 2.1 .

\section{Geometry of Implicit Differential Equations}

For the problem $\mathcal{P}, M$ is a submanifold of $T \mathbb{R}^{n}$; it is obvious that the reduction procedure that we present in the introduction is not applicable to any submanifold $M$ of $T \mathbb{R}^{n}$, especially the submanifolds $M$ for which $\pi_{\mid M}$ admits singularities. In this section we will define the class of submanifolds of $T \mathbb{R}^{n}$ that will be allowed for the problem $\mathcal{P}$. For this class of submanifolds we will be able to apply locally the reduction procedure. First of all, let us make some comparisons with the definition of $\pi$-submanifolds given in [16]. The authors' concern is to answer to the problem of the existence and uniqueness of solutions, namely to put $M$ in the form

$$
M=\phi(Y)
$$

for a section $\phi: Y \rightarrow T Y$ of a connected submanifold $Y$ of $\mathbb{R}^{n}$ with a dimension equal to that of $M$. In this situation, $M$ is equivalent to an ordinary differential equation and, thus, the problem of existence and uniqueness is solved. Here, it is not our purpose to obtain the existence and uniqueness of the solutions (since in this case the optimal control problem admits an obvious solution, namely the trajectory which (possibly) goes from $a$ to $b$ ) but to have the existence and uniqueness of a family of solutions, in other words to find a submanifold $Y$ of $\mathbb{R}^{n}$, an open set $U$ of $\mathbb{R}^{q}$ and a mapping 
$\chi: Y \times U \rightarrow T Y$ such that the diagram

$$
\begin{aligned}
Y \times U & \rightarrow & T Y & \subset T \mathbb{R}^{n} \\
\operatorname{Pr} & \searrow & \downarrow & \pi \\
& & Y & \subset \mathbb{R}^{n}
\end{aligned}
$$

switches and such that

$$
M=\chi(Y \times U) .
$$

This occurs for the submanifold $C(M)$ in the example of the controlled rigid pendulum. Even though in [16] the equality (3.1) suggest that the submanifold $M$ is locally embedded in a tangent bundle $T Y$ of a submanifold $Y$ of $\mathbb{R}^{n}$ with the same dimension as that $M$, in our situation the equality (3.2) suggests that the submanifold $M$ is locally embedded in a tangent bundle $T Y$ of a submanifold $Y$ of $\mathbb{R}^{n}$ of dimension less than or equal to the dimension of $M$.

\section{1. $q-\pi$-SUBMANIFOLD}

For our geometric framework we will consider separable, Haussdorf manifold $X$ with finite dimension and, for reasons of convenience, they are assumed to be smooth (although they could be of class $C^{k}, k \geq 2$ ). Let us recall some elements of differential geometry. The dimension of a manifold $M$ is the maximal dimension among the dimension of the connected components $\Xi$ of $M$. A pure manifold $M$ is a manifold such that all the connected components $\Xi$ have the same dimension. For any manifold $X$, the points belonging to the tangent bundle $T X$ are denoted by $(x, p)$ with $x$ belonging to $X$ and $p$ belonging to $T_{x} X$. The canonical projection $\pi: T X \rightarrow X$ is the mapping such that $\pi(x, p)=x$. For the manifold $\mathbb{R}^{n}$, the tangent bundle is identified with $\mathbb{R}^{n} \times \mathbb{R}^{n}$ and the projection $\pi$ is identified with the projection onto the first factor. Moreover, for any submanifold $Y$ of $X$ and any point belonging to $Y$, the subspace $T_{x} Y$ is identified with a subspace of $T_{x} X$ and, thus, $T Y$ is identified with a submanifold of $T X$. Subsequently, the following notation $f:(X, a) \rightarrow(Y, b)$ means that the mapping $f$ is defined in an open neighborhood $U$ of $a$ in $X$ and $b=f(a)$. As in the case of manifolds, all the mappings are assumed to be smooth (once again they could be of class $C^{k}, k \geq 2$ ). For any mapping $f: X \rightarrow Y$ and any point $x$ belonging to $X$ the linear tangent mapping is denoted by $T_{x} f$. Now let us give the definition of subimmersion and the subimmersion theorem

Definition 3.1. (subimmersion) Let $X, Y$ be manifolds and a mapping $f: X \rightarrow Y$.

(a): $f$ is a subimmersion at $x \in X$ if $r=\operatorname{rank} T_{x} f$ is constant in an open neighborhood of $x$ in $X$.

(b): $f$ is a subimmersion on $X$ if it is a subimmersion at $x$ for all points $x$ of $X$. In particular, for each connected component $\Xi$ of $X$ the rank $r$ has a constant value on $\Xi$, we shall call it the rank of $f$ on $\Xi$.

THEOREM 3.2. (subimmersion theorem) Let $X$ be connected manifolds and $f: X \rightarrow Y$ a subimmersion with rank $r$. Then, the following statements hold

ESAIM: COCV, MaRCH 1998, VOL. 3, 49-81 
(a): for any $y$ belonging to $f(X)$ the set $M=f^{-1}(y)$ is a submanifold of dimension $m-r$ and $T_{x} M=\operatorname{Ker} T_{x} f$.

(b): for any point $x$ belonging to $X$ there exists an open neighborhood $V$ of $x$ in $X$ such that the set $W=f(V)$ is a submanifold of dimension $r$ and $T_{y} W=I m T_{x} f$ for any point $y$ belonging to $W$. Moreover, if $N$ is any submanifold of $X$ of dimension $r$ such that $x$ belongs to $N$ and $T_{x} N \cap \operatorname{ker} T_{x} f=\{0\}$ then the mapping $f_{\mid N}$ is a local diffeomorphism of some open neighborhood of $x$ in $N$ onto an open neighborhood of $y$ in $f(V)$.

Proof. see [9]

For an implicit differential equation (1.1) the following proposition gives a criterion for the projection $\pi_{\mid M}$ to be a subimmersion.

Proposition 3.3. Let $G: \mathbb{R}^{n} \times \mathbb{R}^{n} \rightarrow \mathbb{R}^{n-q}$ be a mapping with $0 \leq q \leq n$ such that $D G(x, p)$ has full rank $n-q$ in an open neighborhood of a point $\left(x_{0}, p_{0}\right)$ belonging to $G^{-1}(0)$ and $U$ an open neighborhood of this point in $\mathbb{R}^{n} \times \mathbb{R}^{n}$ such that the set $M=U \cap G^{-1}(0)$ is a submanifold of $\mathbb{R}^{n} \times \mathbb{R}^{n}$ of dimension $n+q$. Then, the mapping $\pi_{\mid M}$ where $\pi$ is the projection onto the first factor is a subimmersion at $\left(x_{0}, p_{0}\right)$ of rank $r=\rho+q$ if, and only if, rank $D_{p} G(x, p)=\rho \leq n-q$ is constant in an open neighborhood of $\left(x_{0}, p_{0}\right)$ in $M$.

Proof. On the one hand, for any point $(x, p)$ belonging to $M$, the tangent space $T_{(x, p)} M$ is equal to ker $D G(x, p)$ and his dimension is equal to $n+q$. On the other hand, the linear tangent mapping $T_{(x, p)}\left(\pi_{\mid M}\right): T_{(x, p)} M \rightarrow T_{x} \mathbb{R}^{n}$ is the restriction of the canonical projection to the subspace $T_{(x, p)} M$. Namely, the mapping

$$
(\delta x, \delta p) \in T_{(x, p)} M \mapsto \delta x .
$$

Then, for any point $(x, p)$ belonging to $M$

$$
\operatorname{ker} T_{(x, p)}\left(\pi_{\mid M}\right)=\left\{(\delta x, \delta p) \in T_{(x, p)} M / \delta x=0\right\}=\{0\} \times \operatorname{ker} D_{p} G(x, p) .
$$

Clearly, the mapping $\pi_{\mid M}$ has constant rank $r$ in an open neighborhood of $\left(x_{0}, p_{0}\right)$ in $M$ if, and only if, $\operatorname{dim} \operatorname{Im} T_{(x, p)}\left(\pi_{\mid M}\right)=r$ in an open neighborhood of $\left(x_{0}, p_{0}\right)$ in $M$; therefore if, and only if,

$$
\operatorname{dim} \operatorname{ker} T_{(x, p)}\left(\pi_{\mid M}\right)=\operatorname{dim} T_{(x, p)} M-\operatorname{dim} \operatorname{Im} T_{(x, p)}\left(\pi_{\mid M}\right)=n+q-r
$$

in an open neighborhood of $\left(x_{0}, p_{0}\right)$ in $M$. According to $(3.3),(3.4)$ holds if, and only if, $\operatorname{dim} \operatorname{ker} D_{p} G(x, p)=n+q-r=n-\rho$ in an open neighborhood of $\left(x_{0}, p_{0}\right)$ in $M$; that is if, and only if, $\operatorname{dim} \operatorname{Im} D_{p} G(x, p)=\rho$ in an open neighborhood of $\left(x_{0}, p_{0}\right)$ in $M$.

Now we give the definition of a $q$ - $\pi$-submanifold $M$ of $T X$.

Definition 3.4. ( $q-\pi$-submanifold) Let $X$ be a manifold, $q$ a fixed integer less than or equal to the dimension of $X, M$ a submanifold of $T X$ and $(x, p)$ a point of $M . M$ is a $q-\pi-s u b m a n i f o l d ~ o f ~ T X$ at $(x, p)$ (in an neighborhood of $(x, p)$ in $M)$ if the following conditions hold 
(a): there exists a connected open neighborhood $U$ of $(x, p)$ in $M$ and a submanifold $Y$ of $X$ such that $\operatorname{dim} Y+q=\operatorname{dim} U$ and $U$ is a subset of $T Y$.

(b): the mapping $\pi_{\mid U}: U \rightarrow X$ is a subimmersion in the neighborhood of $(x, p)$.

$M$ is a $q-\pi$-submanifold of $T X$ if for any point $(x, p)$ belonging to $M, M$ is a $q$ - $\pi$-submanifold at $(x, p)$.

REMARK 3.5. a) If $M$ is a $q$ - $\pi$-submanifold at a point $(x, p)$ of $M$, then we can assume that the mapping $\pi_{\mid U}: U \rightarrow X$ is a subimmersion on $U$, even if this means shrinking $U$. Moreover, for any point $(x, p)$ belonging to $U$, the first condition of the definition holds (It is enough to take $U$ and $Y$ ). Thus, for any point $(x, p)$ belonging to $U, U$ is a $q$ - $\pi$-submanifold at $(x, p)$; in other words $U$ is a $q-\pi$-submanifold of $T X$.

b) When $M$ is not a $q-\pi$-submanifold of $T X$ we can consider the set, possibly empty, of points $(x, p)$ of $M$ such that $M$ is $q-\pi$-submanifold of $T X$ at $(x, p)$. If it is a non-empty set, according to a), it is an open set of $M$ and a $q-\pi$ submanifold of $T X$.

The definition of a $q-\pi$-submanifold can be formulated in the following way

Definition 3.6. (bis) Let $X$ be a manifold and $q$ an integer less than or equal to the dimension of $X$. A submanifold $M$ of $T X$ is a $q-\pi$-submanifold of $T X$ if for any connected component $\Xi$ of $M$ the following conditions hold.

(a): for any point $(x, p)$ of $\Xi$ there exists an open neighborhood $U$ in $\Xi$ of $(x, p)$ and a submanifold $Y$ of $X$ such that $\operatorname{dim} Y+q=\operatorname{dim} \Xi$ and $U$ is a subset of $T Y$.

(b): the mapping $\pi_{\mid \Xi}: \Xi \rightarrow X$ is a subimmersion in a neighborhood of any point $(x, p)$ of $\Xi$.

REMARK 3.7. This definition extends the definition of a $\pi$-submanifold in [16], which is the case $q=0$ of our definition. The first condition means exactly that $M$ is locally embedded in the tangent bundle $T Y$ of a submanifold $Y$ of $X$ of dimension less than or equal to the dimension of $M$. For the second, according to the subimmersion theorem, for any point $(x, p)$ of $M$ there exists an open neighborhood $V$ of $(x, p)$ in $M$ such that $W=\pi(V)$ is a submanifold of $X$ of dimension the rank of the mapping $\pi_{\mid \Xi}$ at $(x, p)$. This is the local analogous of the condition, $W=\pi(M)$ is a submanifold of $X$, supposed in the global reduction procedure. For any connected component $\Xi$ of $M$ the inequality $2 q \leq \operatorname{dim} \Xi$ is satisfied $(\operatorname{dim} \Xi \leq 2 \operatorname{dim} Y$, $\operatorname{dim} Y+q=\operatorname{dim} \Xi$ ). We shall use this inequality to prove a property of the index.

EXAMPLE 3.8. Let $\chi: X \times U \rightarrow T X$ be a smooth controlled vector field with $\operatorname{dim} U=q$. Let us assume that

(a): the mapping $\frac{\partial \chi}{\partial u}(x, u)$ has full rank $q$.

(b): for any $(x, p)$ belonging to $T X$ either the equation $\chi(x, u)=(x, p)$

has a unique solution or it does not have any solution.

(c): the mapping $(x, u) \mapsto \chi(x, u)$ is proper.

ESAIM: COCV, MARCH 1998, VOL. 3, 49-81 
Clearly, under this mild assumption the set $M=\chi(X \times U)$ is a submanifold of $T X^{3}$. Obviously, $\operatorname{dim} M=\operatorname{dim} X+\operatorname{dim} U$ and $\pi_{\mid M}$ is a subimmersion. Thus, $M$ is a $q$ - $\pi$-submanifold.

In the example of the controlled rigid pendulum, the submanifold $M_{0}$ of $T \mathbb{R}^{7}$ is connected and its dimension is equal to 7 . Therefore, for any point $(x, p)$ belonging to $M_{0}$ the first condition holds with $U=M_{0}, Y=\mathbb{R}^{7}$ and $q=2$. Moreover, for any point $(x, p)$ belonging to $M_{0}$, rank $D_{p} G(x, p)=4$; then, according to the proposition 3.3, the mapping $\pi_{\mid M_{0}}$ is a subimmersion of rank $4+2=6$. Therefore, the submanifold $M_{0}$ is a $2-\pi$-submanifold of $T \mathbb{R}^{7}$.

Now we shall give some definitions: the order of point $(x, p)$ of $M$ is the rank of the mapping $\pi_{\mid \Xi}$ at this point, we shall denote it by ord ${ }_{M}(x, p)$. Since, the mapping $\pi_{\mid \Xi}$ has, locally, constant rank, ord ${ }_{M}(x, p)$ is constant for each point of any connected component of $M$, then we may define ord ${ }_{M} \Xi$ as the order of one of its points and it is less than or equal to the dimension of the submanifold $Y$. A submanifold $W$ as in the remark 3.7 is called a local projection of $M$ at $(x, p)$.

REMARK 3.9. With the notations of the definition of a $q-\pi$-submanifold, $U$ is a submanifold of $T Y$ and since the mapping $\pi_{\mid \Xi}: \Xi \rightarrow X$ is a subimmersion, the mapping $\pi_{\mid U}: U \rightarrow X$ is a subimmersion. This is satisfied if, and only if, the mapping $\pi_{\mid U}: U \rightarrow Y$ is a subimmersion. The order of a point belonging to $\Xi$ is also the rank of the mapping $\pi_{\mid U}: U \rightarrow Y$ at this point. Then, we can see $U$ as a submanifold of $T Y$ and as a submanifold of $T X$.

The following theorem ensures that a $q-\pi$-submanifold $M$ is, locally, the image of a unique controlled vector field.

Theorem 3.10. (Existence and uniqueness) Given $X$ a manifold and $M a$ $q-\pi$-submanifold of $T X$ such that

$$
\operatorname{dim} \Xi=\text { ord }_{M} \Xi+q
$$

for each connected component $\Xi$ of $M$, then for each point $(x, p)$ of $M$ there exists a local projection $W=\pi(V)$ of $M$ at $(x, p)$, an open set $O$ of $\mathbb{R}^{q}$ and a unique smooth mapping $\chi: W \times O \rightarrow T W$ such that

$$
V=\chi(W \times O), p=\chi(x, 0), \operatorname{rank} \frac{\partial \chi}{\partial u}(x, 0)=q
$$

and such that $\operatorname{Pr}=\pi \circ \times$ where $P r$ is the canonical projection from $W \times O$ onto $W$. Moreover, if $W^{\prime}=\pi\left(V^{\prime}\right)$ is another local projection of $M$ at $(x, p)$ such that there exists an open set $O^{\prime}$ of $\mathbb{R}^{q}$ and a unique smooth mapping $\chi^{\prime}: W^{\prime} \times O^{\prime} \rightarrow T W^{\prime}$ such that

$$
V^{\prime}=\chi\left(W^{\prime} \times O^{\prime}\right), p=\chi^{\prime}(x, 0), \operatorname{rank} \frac{\partial \chi^{\prime}}{\partial u^{\prime}}(x, 0)=q
$$

and such that $\mathrm{Pr}^{\prime}=\pi \circ \chi^{\prime}$ where $P r^{\prime}$ is the canonical projection from $W^{\prime} \times O^{\prime}$ onto $W^{\prime}$, then there exists a diffeomorphism $h:(W \times O,(x, 0)) \rightarrow\left(W^{\prime} \times\right.$ $\left.O^{\prime},(x, 0)\right)$ such that $\chi=\chi^{\prime} \circ h$ and $P r=P r^{\prime} \circ h$.

Proof. Let $\left(x_{0}, p_{0}\right)$ be a point of $M$. According to (3.5) and the remark 3.9 the mapping $\pi_{\mid U}: U \rightarrow Y$ is a subimmersion with rank equal to Ord ${ }_{M} \Xi=$

\footnotetext{
${ }^{3}$ Since the mapping $\chi$ is an injective proper immersion.
} 
$\operatorname{dim} \Xi-q=\operatorname{dim} Y$, therefore it is a submersion. Then, there exists an open neighborhood $V$ of $\left(x_{0}, p_{0}\right)$ in $U$ such that the local projection $W=\pi(V)$ is an open set of $Y$. Since $W$ is an open set of $Y$, the tangent bundle $T W$ of $W$ is equal to $\left(\pi_{\mid T Y}\right)^{-1}(W)=\pi^{-1}(W) \cap T Y$. Moreover, $V \subset T Y$ and $V \subset$ $\pi^{-1}(W)$ then $V \subset \pi^{-1}(W) \cap T Y=T W$. Then, $V$ is a submanifold of $T W$ of $\operatorname{dimension} \operatorname{dim} Y+q$. This last property is also satisfied when we shrink $V$ or $W$ (for any open set $V^{\prime}$ of $V, W^{\prime}=\pi\left(V^{\prime}\right)$ is also a local projection and $V^{\prime}$ is a submanifold of $T W^{\prime}$ by the same arguments; for any open set $W^{\prime}$ of $W$ setting $V^{\prime}=\left(\pi_{\mid T Y}\right)^{-1}\left(W^{\prime}\right) \cap V$ then $W^{\prime}=\pi\left(V^{\prime}\right)$ and $V^{\prime}$ is also a submanifold of $\left.T W^{\prime}\right)$. Thus, even if this means shrinking $V$, we can assume that there exists a chart $(W, \psi)$ of $Y$ such that $W$ is identified with an open set of $\mathbb{R}^{m}$, also denoted by $W$, then $T W$ is identified with $W \times \mathbb{R}^{m}, \pi$ is identified with the canonical projection and $V$ is identified with a submanifold of $W \times \mathbb{R}^{m}$ projected onto $W$. We can also assume that there exists an open set $\Omega$ of $\left(x_{0}, p_{0}\right)$ in $W \times \mathbb{R}^{m}$, an open set $\Omega_{v}$ of 0 in $\mathbb{R}^{m-q}$ and a submersion $G: \Omega \rightarrow \Omega_{v}$ such that $V \cap \Omega=G^{-1}(0) \cap \Omega$. Since $V$ is projected onto $W$ then $D_{p} G\left(x_{0}, p_{0}\right)$ has full rank; ; even if this means shrinking $\Omega$, there exists an open set $\Omega_{u}$ of 0 in $\mathbb{R}^{q}$ and a mapping $H: \Omega \rightarrow \Omega_{u}$ such that for the mapping $\Phi: \Omega \rightarrow W \times \Omega_{u} \times \Omega_{v}$ defined by $(y, u, v)=\Phi(x, p)=(x, H(x, p), G(x, p))$, $D \Phi\left(x_{0}, p_{0}\right)$ is an isomorphism. According to the local inverse functions theorem there exists an open set $\Omega^{\prime} \subset \Omega$ of $\left(x_{0}, p_{0}\right)$ in $W \times \mathbb{R}^{m}$ and an open set $\Omega^{\prime \prime}$ of $\left(x_{0}, 0,0\right)$ in $W \times \Omega_{u} \times \Omega_{v}$ such that $\Phi_{\mid \Omega^{\prime}}: \Omega^{\prime} \rightarrow \Omega^{\prime \prime}$ is a diffeomorphism. We can assume that $\Omega^{\prime \prime}$ has the form $W^{\prime} \times \Omega_{u}^{\prime} \times \Omega_{v}^{\prime}$ where $W^{\prime}$ is an open set of $W, \Omega_{u}^{\prime}$ is an open set of $\Omega_{u}$ and $\Omega_{v}^{\prime}$ is an open set of $\Omega_{v}$. Moreover there exists an open set $\Omega_{p}^{\prime}$ of $p$ in $\mathbb{R}^{m}$ such that $\Omega^{\prime}=\Phi_{\mid \Omega^{\prime}}^{-1}\left(\Omega^{\prime \prime}\right)$ has the form $W^{\prime} \times \Omega_{p}^{\prime}$. Let $V_{0}=V \cap \Omega^{\prime}$, then $W_{0}=\pi\left(V_{0}\right)$ is a local projection of $M$ at $\left(x_{0}, p_{0}\right)$. Given $\Phi_{\mid \Omega^{\prime}}^{-1}(y, u, v)=(y, \phi(y, u, v))$ the inverse mapping of $\Phi_{\mid \Omega^{\prime}}$, then $V_{0}=\left\{\left(x, \phi(x, u, 0), x \in W_{0}, u \in \Omega_{u}^{\prime}\right\}=\Phi_{\mid \Omega^{\prime}}^{-1}\left(W_{0} \times \Omega_{u}^{\prime} \times\{0\}\right)\right.$. Therefore, we define $O=\Omega_{u}^{\prime} \times\{0\}$ and $\chi=\Phi_{\mid W_{0} \times O}^{-1}$. Then $V_{0}=\chi\left(W_{0} \times O\right)$, $\chi\left(x_{0}, 0\right)=p_{0}$ and $\operatorname{rank} \frac{\partial \chi}{\partial u}\left(x_{0}, 0\right)=\operatorname{rank} \frac{\partial \phi}{\partial u}\left(x_{0}, 0,0\right)=q$. Given $W^{\prime}=\pi\left(V^{\prime}\right)$ another local projection of $M$ at $(x, p)$ such that there exists an open set $O^{\prime}$ of $\mathbb{R}^{q}$ and a unique smooth mapping $\chi^{\prime}: W^{\prime} \times O^{\prime} \rightarrow T W^{\prime}$ such that $V^{\prime}=\chi\left(W^{\prime} \times O^{\prime}\right), p=\chi^{\prime}(x, 0), \operatorname{rank} \frac{\partial \chi^{\prime}}{\partial u^{\prime}}(x, 0)=q$ and such that $P r^{\prime}=\pi \circ \chi^{\prime}$ where $\mathrm{Pr}^{\prime}$ is the canonical projection from $W^{\prime} \times O^{\prime}$ onto $W^{\prime}$, then the implicit equation $\chi^{\prime}\left(x, u^{\prime}\right)=\chi(x, u)$ is locally invertible relative to $u$ and $u^{\prime}$ and the existence of $h$ follows.

REMARK 3.11. The diagrams

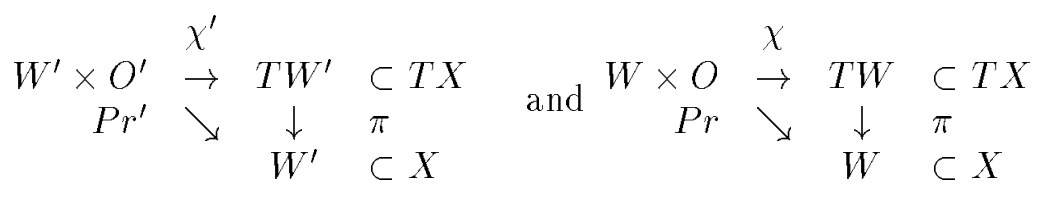

are equivalent in a neighborhood of $(x, 0)$ by the bundle isomorphism $h$. Such bundle isomorphisms define in control theory a feedback. 


\subsection{REDUCTION OF A $q-\pi$-SUBMANIFOLD}

Since the condition of the theorem 3.10 is generally not fulfilled, then we have to define the reduction procedure. In the case of the global reduction procedure, we have seen that any admissible trajectory $x(\cdot)$ for $M_{0}$ is necessarily an admissible trajectory for $M_{1}=M_{0} \cap T W_{0}$ and $M_{1}$ is the reduction of $M_{0}$. In order to have, locally, the same reduction procedure, we use local projection. Let $x(\cdot)$ be a local admissible trajectory, in other words $(x(\cdot), \dot{x}(\cdot))$ is a trajectory of $M$ passing through the point $\left(x_{0}, p_{0}\right)=\left(x\left(t_{0}\right), \dot{x}\left(t_{0}\right)\right) \in M$ at time $t_{0}$, where $t_{0}$ is a point of continuity; since $M$ is a $q-\pi$-submanifold, then for a local projection $W=\pi(V)$ at $\left(x_{0}, p_{0}\right)$ of $M$ and for $t$ in a neighborhood of $t_{0},(x(t), \dot{x}(t)) \in V$, whence $x(t) \in W=\pi(V)$ and $(x(t), \dot{x}(t)) \in T W$. In particular $\left(x_{0}, p_{0}\right) \in T W \cap V \subset T W \cap M$. This leads to the following definition

Definition 3.12. Let $X$ be a manifold and $M$ a $q-\pi$-submanifold of $T X$. A point $(x, p) \in M$ is a point of reducibility of $M$ if there exists a local projection $W=\pi(V)$ of $M$ at $X$ such that $p \in T_{x} W$, in other words $(x, p)$ belongs to $T W \cap M$. The (possibly empty) set of the points of reducibility of $M$ is the reduction of $M$, we denote it by $M^{\prime}$

REMARK 3.13. If such local projection $W=\pi(V)$ exists then for any other local projection $W^{\prime}=\pi\left(V^{\prime}\right)$ of $M$ at $(x, p)$, since $T_{x} W^{\prime}=T_{x} W,(x, p)$ belongs to $T W^{\prime} \cap M$. Thus, the definition does not depend on the choice of the local projection $W$.

Example 3.14. A $q-\pi$-submanifold $M$ such that the reduction $M^{\prime}$ is the empty set.

In $T \mathbb{R}^{5}$ let $M$ be the submanifold given by the implicit differential equation $F_{0}=0$ where

$$
F_{0}(x, p)=\left(\begin{array}{c}
x_{1}-x_{2} \\
p_{1}-1 \\
p_{2} \\
p_{3}-p_{4}-p_{5}
\end{array}\right)
$$

Clearly, $M$ is a $1-\pi$-submanifold of $T \mathbb{R}^{5}, W=\pi(M)$ is the submanifold of dimension 4 of $\mathbb{R}^{5}$ given by the equation $x_{1}-x_{2}=0$ and $M^{\prime}=T W \cap M=\emptyset$.

Now that we have stated the reduction procedure and a new set, namely $M^{\prime}$ the reduction of $M$, we are going to see under which conditions for $M$, the reduction $M^{\prime}$ is a submanifold and a $q-\pi$-submanifold. First of all, we can establish the following results

Proposition 3.15. Let $X$ be a manifold and $M$ a $q-\pi$-submanifold of $T X$. Then, the reduction $M^{\prime}$ of $M$ is a closed subset of $M$.

Proof. The cases $M=M^{\prime}$ and $M=\emptyset$ are obvious. Let us assume that $\emptyset \nsubseteq M^{\prime} \nsubseteq M$ and let us consider the subset $N=M \backslash M^{\prime}$. Let $(x, p)$ be a point of $N$ and $W=\pi(V)$ a local projection of $M$ at this point. $W$ is a submanifold of $X$ of dimension $r=$ ord $_{M} \Xi$. Upon shrinking $V$, we can assume that there exists a chart $(\Omega, \phi)$ of $X$ such that $W \subset \Omega, \phi(\Omega)$ is an open set of $\mathbb{R}^{n}, \phi(W)$ is an open set of $\mathbb{R}^{r}, T \Omega$ is identified with $\Omega \times \mathbb{R}^{n}$, $T W$ is identified with $W \times \mathbb{R}^{r}$ and $V$ is identified with a submanifold of $\Omega \times \mathbb{R}^{n}$. Since, the point $(x, p)$ belongs to $N$ and the point $x$ belongs to $W$ 
the point $p$ does not belong to $T_{x} W$, and in the chart this means that $p$ does not belong to $\mathbb{R}^{r}$. Thus, there exists an open neighborhood $V^{\prime}$ of $(x, p)$ in $V$ such that $V^{\prime} \cap T W=\emptyset$. Therefore, the set $N$ is an open set of $M$ and then $M^{\prime}$ is a closed subset of $M$.

The following proposition is used to establish the conditions such that the reduction $M^{\prime}$ of a $q-\pi$-submanifold $M$ is a submanifold.

Proposition 3.16. Let $X$ be a manifold and $M$ a $q-\pi$-submanifold of $T X$ such that its reduction $M^{\prime}$ is not empty. Given $(x, p)$ a point of $M^{\prime}$, then for any local projection $W=\pi(V)$ of $M$ at $(x, p)$

$$
\operatorname{dim}\left[T_{(x, p)} T W \cap T_{(x, p)} M\right] \geq \operatorname{ord}_{M}(x, p)+q .
$$

Proof. Let $\left(x_{0}, p_{0}\right)$ be a point of $M^{\prime}$ and $W=\pi(V)$ a local projection of $M$ at $\left(x_{0}, p_{0}\right)$. According to the definition of a $q$ - $\pi$-submanifold there exists an open neighborhood $U$ of $\left(x_{0}, p_{0}\right)$ in $M$ and a submanifold $Y$ of $X$ such that $\operatorname{dim} Y+q=\operatorname{dim} \Xi$ and $U$ is a subset of $T Y$. We can choose $W=\pi(V)$ such that $V \subset U$, therefore $W \subset \pi(U) \subset Y$. Let $i: W \rightarrow Y$ be the canonical embedding of $W$ in $Y$ and

$$
\Sigma=i^{\star} T Y=\bigcup_{x \in W}\{x\} \times T_{x} Y \subset T Y .
$$

$\Sigma$ is a submanifold of $T Y$ of dimension equal to the sum of the dimension of $W$ and of the dimension of $Y$. Moreover, $T W$ and $V$ are submanifold of $\Sigma$. Indeed, let $(x, p)$ be a point of $\Sigma$. Then, according to the construction of $\Sigma, x$ belongs to $W$ and $p$ belongs to $T_{x} Y$. Since $Y$ and $W$ are submanifolds of $X$, there exists in a neighborhood of the point $x$ in $X$ two submersions $g:\left(\mathbb{R}^{n}, x\right) \rightarrow\left(\mathbb{R}^{n-r}, 0\right)$ and $h:\left(\mathbb{R}^{n}, x\right) \rightarrow\left(\mathbb{R}^{n-m}, 0\right)$ such that in a neighborhood of $x, W=g^{-1}(0)$ and $Y=h^{-1}(0)$ where $m=\operatorname{dim} Y=\operatorname{dim} \Xi-q$ and $r=$ ord $M(x, p)=$ ord ${ }_{M}\left(x_{0}, p_{0}\right)$. Thus in a neighborhood of $(x, p)$ in $T X, \Sigma$ is the zero set of the submersion

$$
\begin{gathered}
\sigma:(T X,(x, p)) \rightarrow\left(\mathbb{R}^{n-r} \times \mathbb{R}^{n-m},(0,0)\right) \\
(x, p) \mapsto \sigma(x, p)=(g(x), d h(x) \cdot p) .
\end{gathered}
$$

Thus, $\Sigma$ is a submanifold of $T X$ of dimension $n-r-(n-m)=r+m$ and therefore a submanifold of $T Y$. According to the construction, $T W$ is a subset of $\Sigma$ and a submanifold of $T X$, therefore $T W$ is a submanifold of $\Sigma$. Finally, since $V$ is subset of $\Sigma$ (for any point $(x, p)$ of $V \subset U \subset T Y, x$ belongs to $W$ and $p$ belongs to $T_{x} Y$ ) and a submanifold of $T Y, V$ is a submanifold of $\Sigma$. Since $\operatorname{dim} V=\operatorname{dim} Y+q, \operatorname{dim} T W=2 r$ and $\operatorname{dim} \Sigma=\operatorname{dim} Y+r$ then

$$
\begin{aligned}
\operatorname{dim}\left[T_{(x, p)} T W \cap T_{(x, p)} V\right] & \geq \operatorname{dim} T_{(x, p)} T W+\operatorname{dim} T_{(x, p)} V-\operatorname{dim} T_{(x, p)} \Sigma \\
& =\operatorname{ord}{ }_{M}(x, p)+q .
\end{aligned}
$$

TheOREM 3.17. Let $X$ be a manifold and $M$ a $q-\pi$-submanifold of $T X$ such that its reduction $M^{\prime}$ is not empty. If for each point $(x, p)$ of the reduction $M^{\prime}$ of $M$ there exists a local projection $W=\pi(V)$ of $M$ at $(x, p)$ such that

$$
\operatorname{dim}\left[T_{(x, p)} T W \cap T_{(x, p)} M\right]=\operatorname{ord}_{M}(x, p)+q,
$$


then $M^{\prime}$ is a submanifold and $T_{(x, p)} M^{\prime}=T_{(x, p)} T W \cap T_{(x, p)} M$. Moreover, if in an open neighborhood of $(x, p)$ in $T W \cap M$

$$
\pi_{\mid T_{\left(x^{\prime}, p^{\prime}\right)} M^{\prime}} \text { has constant rank, }
$$

then for any connected component $\Xi$ of $M, \Xi^{\prime}$ the reduction of $\Xi$ is either empty or a pure $q-\pi$-submanifold of dimension ord $M \Xi+q$. In particular $M^{\prime}$ is a $q-\pi-$ submanifold.

Proof. Given $(x, p)$ a point of $M^{\prime}$ and $W=\pi(V)$ a local projection of $M$ at $(x, p)$ such that $(3.6)$ is satisfied, then the submanifolds $T W$ and $V$ are transversal in the bundle $\Sigma$ at $(x, p)$, therefore $T W \cap V$ is a submanifold of $T W$ and of $M$ of dimension ord ${ }_{M}(x, p)+q$. The mapping $\pi_{\mid V}$ has constant rank on $V$, even if this means shrinking $V$. But, on the one hand,

$M^{\prime} \cap V=\left\{(x, p) \in V / \exists\right.$ a local projection $\left.W_{(x, p)}=\pi\left(V_{(x, p)}\right) / p \in T_{x} W_{(x, p)}\right\}$ and, on the other hand, $W=\pi(V)$ is a local projection of $M$ at each points of $V$, therefore

$$
M^{\prime} \cap V=\left\{(x, p) \in V / p \in T_{x} W\right\}=T W \cap V .
$$

In other words $M^{\prime}$ is a submanifold of $M$ and $T_{(x, p)} M^{\prime}=T_{(x, p)} T W \cap$ $T_{(x, p)} V=T_{(x, p)} T W \cap T_{(x, p)} M$.

Therefore the connected component of $M^{\prime}$ containing the point $(x, p)$ has a dimension equal to ord $M(x, p)+q$. Let us prove that the first condition of the definition of a $q-\pi$-submanifold hold. Given $\Xi^{\prime}$ the connected component of $M^{\prime}$ containing $(x, p)$, there exists an open neighborhood $U^{\prime}$ of $(x, p)$ in $\Xi^{\prime}$ such that $U^{\prime} \subset T W$, in fact $U^{\prime}=T W \cap V$ that we can assume included in $\Xi^{\prime}$. If we set down $Y^{\prime}=W$ then $\operatorname{dim} Y^{\prime}+q=\operatorname{dim} \Xi^{\prime}$ and the condition holds. Moreover, if condition (3.7) holds then $\pi_{\mid \Xi}$ has constant rank and therefore according to the previous results, $M^{\prime}$ is a $q-\pi$-submanifold of $X$.

The theorem 3.17 justified the following definition

Definition 3.18. Let $X$ be a manifold and $M$ a $q-\pi$-submanifold $M$ of $T X$ such that its reduction $M^{\prime}$ is not empty. $M$ is a reducible $q-\pi$-submanifold if for any point $(x, p)$ of the reduction $M^{\prime}$ of $M$ there exists a local projection $W=\pi(V)$ of $M$ at $(x, p)$ such that

$$
\operatorname{dim}\left[T_{(x, p)} T W \cap T_{(x, p)} M\right]=\text { ord }{ }_{M}(x, p)+q
$$

and if in an open neighborhood of $(x, p)$ in $T W \cap M$

$$
\pi_{\mid T_{\left(x^{\prime}, p^{\prime}\right)} M^{\prime}} \text { has constant rank }
$$

where $T_{\left(x^{\prime}, p^{\prime}\right)} M^{\prime}=T_{\left(x^{\prime}, p^{\prime}\right)} T W \cap T_{\left(x^{\prime}, p^{\prime}\right)} M$.

REMARK 3.19. If $M$ is a reducible $q-\pi$-submanifold of class $c^{k}, k \geq 2$, then its reduction is a $q-\pi$-submanifold of class $C^{k-1}$.

EXAMPLE 3.20. q- $\pi$-submanifold $M$ such that the reduction $M^{\prime}$ is not empty

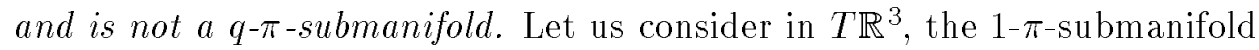
given by the implicit differential equation $F_{0}=0$ where

$$
F_{0}(x, p)=\left(\begin{array}{c}
x_{1} \\
p_{1}+x_{2}+p_{3}^{2}
\end{array}\right) .
$$


Clearly, $W=\pi(M)$ is the submanifold of dimension 2 of $\mathbb{R}^{3}$ given by the equation $x_{1}=0$. Its reduction $M_{1}$ is equal to $F_{1}^{-1}(0)$ where

$$
F_{1}(x, p)=\left(\begin{array}{c}
x_{1} \\
p_{1} \\
x_{2}+p_{3}^{2}
\end{array}\right)
$$

is not a $1-\pi$-submanifold.

We can now provide a new formulation of the theorem 3.10

Theorem 3.21. (Existence and uniqueness) Given $X$ a manifold and $M a$ $q-\pi$-submanifold of $T X$ such that its reduction $M^{\prime}$ is $M$, then for each point $(x, p)$ of $M$ there exists a local projection $W=\pi(V)$ of $M$ at $(x, p)$, an open set $O$ of $\mathbb{R}^{q}$ and a unique smooth mapping $\chi: W \times O \rightarrow T W$ such that

$$
V=\chi(W \times O), p=\chi(x, 0), \operatorname{rank} \frac{\partial \chi}{\partial u}(x, 0)=q
$$

and such that $\operatorname{Pr}=\pi \circ \chi$ where $\operatorname{Pr}$ is the canonical projection from $W \times O$ onto $W$. Moreover, if $W^{\prime}=\pi\left(V^{\prime}\right)$ is another local projection of $M$ at $(x, p)$ such that there exists an open set $O^{\prime}$ of $\mathbb{R}^{q}$ and a unique smooth mapping $\chi^{\prime}: W^{\prime} \times O^{\prime} \rightarrow T W^{\prime}$ such that

$$
V^{\prime}=\chi\left(W^{\prime} \times O^{\prime}\right), p=\chi^{\prime}(x, 0), \operatorname{rank} \frac{\partial \chi^{\prime}}{\partial u^{\prime}}(x, 0)=q
$$

and such that $P r^{\prime}=\pi \circ \chi^{\prime}$ where $P r^{\prime}$ is the canonical projection from $W^{\prime} \times O^{\prime}$ onto $W^{\prime}$, then there exists a diffeomorphism $h:(W \times O,(x, 0)) \rightarrow\left(W^{\prime} \times\right.$ $\left.O^{\prime},(x, 0)\right)$ such that $\chi=\chi^{\prime} \circ h$ and $\operatorname{Pr}=P r^{\prime} \circ h$.

Proof. The connected component $\Xi^{\prime}$ of $M^{\prime}$ is exactly the connected component $\Xi$ of $M$, but according to the theorem $3.17 \mathrm{dim} \Xi=\operatorname{dim} \Xi^{\prime}=$ ord ${ }_{M} \Xi+q$ then assumption (3.5) of the theorem 3.10 holds for any connected component of $M$.

REMARK 3.22. For any $q-\pi$-submanifold $M$ of $T X$ such that for any connected component $\Xi$ of $M$ the equality (3.5) holds then $M$ is equal to its reduction. Clearly, for any point $(x, p)$ of $M$ there exists a local projection $W=\pi(V)$ of $M$ at $(x, p)$ such that $p$ belongs to $T_{x} W \cap M$.

This remark leads to the following proposition

Proposition 3.23. Let $X$ be a manifold, q a fixed integer less than or equal to the dimension of $X$ and $M$ a submanifold of $T X$. Let us assume that for any point $(x, p)$ in $M$ there exists a connected open neighborhood $U$ of $(x, p)$ in $M$ and a submanifold $Y$ of $X$ such that $\operatorname{dim} Y+q=\operatorname{dim} U$ and $U$ is a subset of $T Y$. Then $M$ is a reducible $q-\pi$-submanifold of $T X$ equal to its reduction $M^{\prime}$ if, and only if, for any connected component $\Xi$ of $M$ there exists a point $(x, p)$ in $\Xi$, such that the linear mapping $T_{(x, p)} \pi: T_{(x, p)} M \rightarrow T_{(x, p)} Y$ is surjective.

Proof. If $M$ is a reducible $q-\pi$-submanifold of $T X$ equal to its reduction then for any connected component $\Xi$ and for any point $(x, p)$ of $\Xi$ the linear mapping $T_{(x, p)} \pi: T_{(x, p)} M \rightarrow T_{(x, p)} Y$ is surjective. Clearly, this must hold only for one point $(x, p)$ of any connected component $\Xi$ of $M$. Conversely, let us assume that for any connected component $\Xi$ of $M$ there exists a point $(x, p)$ in $\Xi$ such that the linear mapping $T_{(x, p)} \pi: T_{(x, p)} M \rightarrow T_{(x, p)} Y$ ESAIM: COCV, MARCH 1998, VOL. 3, 49-81 
is surjective. Thus, the mapping $\pi_{\mid U}: U \rightarrow Y$ has full rank $\operatorname{dim} Y$ and according to the remark 3.9 the mapping $\pi_{\mid U}: U \rightarrow X$ has constant rank. Then $M$ is a $q-\pi$-submanifold of $T X$. In particular for each connected component $\Xi$ of $M$ the equality (3.5) is satisfied. Thus, according to the remark $3.22, M$ is equal to its reduction.

\subsection{COMPLETEly ReDUCiBle $q$ - $\pi$-SUBMANifold}

Given a reducible $q$ - $\pi$-submanifold $M$ of a manifold $T X$, then according to the theorem 3.17 its reduction $M_{1}$ is a $q-\pi$-submanifold. Clearly, the reduction $M_{2}$ of $M_{1}$ may be empty (example 3.14 ) and in the case where $M_{2}$ is not empty, $M_{2}$ may not be reducible (example 3.20 ). If $M_{1}$ is reducible then $M_{2}$ is a $q$ - $\pi$-submanifold. Thus, we can, if it is possible, consider the successive reductions of $M$ (example 2.9). For reasons of convenience, we shall say that the empty set is a reducible $q$ - $\pi$-submanifold such that its reduction is the empty set. These considerations lead us to consider the definition of a completely reducible $q$ - $\pi$-submanifold

Definition 3.24. Let $X$ be a manifold and $M$ a $q-\pi$-submanifold of $T X$. We shall say that $M$ is a completely reducible $q-\pi-$ submanifold if it is reducible and if its reduction $M^{\prime}$ is a completely reducible $q$ - $\pi$-submanifold.

The definition means that it is possible to construct a sequence of reducible $q-\pi$-submanifolds $M_{k}, k \geq 0$ such that $M=M_{0}, M_{k+1}=M_{k}^{\prime}$ if $M_{k} \neq \emptyset$ and $M_{k+1}=M_{k}$ if $M_{k}=\emptyset$. This sequence of $q-\pi$-submanifold is called the chain of reduction of $M$. If for an integer $\alpha, M_{\alpha+1}=M_{\alpha}$ then the sequence $M_{k}$ becomes stationary at and after the integer $\alpha$. Since the sequence $\operatorname{dim} M_{k}$ is decreasing, we can expect the chain of reduction of $M$ to be stationary.

Theorem 3.25. (Stationarity) Let $X$ be a manifold and $M$ a completely reducible $q-\pi$-submanifold of $T X, m$ its dimension and $\left\{M_{k}\right\}_{k \geq 0}$ its chain of reduction.

(a): given $\Xi_{m+1-2 q}$ a non-empty connected component of $M_{m+1-2 q}$ and for any $k=0, \cdots, m-2 q, \Xi_{k}$ the connected component of $M_{k}$ containing $\Xi_{k+1}$, then there exists a smallest integer $\alpha, 0 \leq \alpha \leq \operatorname{dim} \Xi_{0}-2 q \leq$ $m-2 q$, such that $\Xi_{k}^{\prime}=\Xi_{k}$ for any $k \geq \alpha$.

(b): the reduction $M_{m+2-2 q}$ of $M_{m+1-2 q}$ is $M_{m+1-2 q}$.

Proof. When $M_{m+1-2 q}=\emptyset$ we do not take $(a)$ into account and with the convention, $(b)$ is obvious. Let us assume that $M_{m+1-2 q}$ is not empty and let us consider $\Xi_{m+1-2 q}$ one of its non empty connected component. For any $k=0, \cdots, m-2 q$ the reduction $\Xi_{k}^{\prime}$ of $\Xi_{k}$ is by definition the set of points of reducibility of $\Xi_{k}$, namely the set of points of reducibility of $M_{k}$ belonging to $\Xi_{k}$ i.e. $\Xi_{k}^{\prime}=\Xi_{k} \cap M_{k+1}$. Thus from the construction of the sequence $\Xi_{k}$, the connected component $\Xi_{k+1}$ of $M_{k+1}$ included in $\Xi_{k}$ is a connected component of the reduction $\Xi_{k}^{\prime}$ of $\Xi_{k}$ and a closed subset of $\Xi_{k}$. Then we have

$$
\Xi_{k+1} \subset \Xi_{k}^{\prime} \subset \Xi_{k}, \quad k=0, m-2 q .
$$

Therefore, the sequence $\nu_{k}=\operatorname{dim} \Xi_{k}, k=0, \cdots, m-2 q$ satisfy

$$
2 q \leq \nu_{m-2 q+1} \leq \nu_{m-2 q} \leq \cdots \leq \nu_{0} \leq m
$$


and thus there exists a smallest integer $\alpha$ between 0 and $\nu_{0}-2 q$ such that $\nu_{\alpha}=\nu_{\alpha+1}$. Thus $\Xi_{\alpha+1}$ is an open set of $\Xi_{\alpha}$. Since $\Xi_{\alpha+1}$ is also a non empty closed subset of $\Xi_{\alpha}$ then $\Xi_{\alpha}=\Xi_{\alpha+1}$. According to (3.10) the reduction $\Xi_{\alpha}^{\prime}$ of $\Xi_{\alpha}$ is exactly $\Xi_{\alpha+1}$ and therefore, for any $k=\alpha, \cdots, 2 m-q+1$, the reduction $\Xi_{k}^{\prime}$ of $\Xi_{k}$ is $\Xi_{k+1}$. Finally, the reduction $\Xi_{m+1-2 q}^{\prime}$ of any connected component $\Xi_{m+1-2 q}$ of $M_{m+1-2 q}$ is $\Xi_{m+2-2 q}$, thus $M_{m+1-2 q}=$ $M_{m+2-2 q}$.

Definition 3.26. (Index) Let $X$ be a manifold, $M$ a completely reducible $q-\pi$-submanifold of $T X, m$ the dimension of, and $\left\{M_{k}\right\}_{k>0}$ the chain of reduction of $M$.

(a): the core of the completely reducible $q-\pi$-submanifold $M$ is the limit of its chain of reduction and we denote it by $C(M)$.

(b): the index of any non-empty connected component $\Xi$ of $C(M)$ is the integer $\alpha$ in $3.25(\mathrm{a})$.

(c): the index of any point $(x, p)$ of $C(M)$ is the index of the connected component of $C(M)$ containing $(x, p)$. In particular it is less than or equal to $\operatorname{dim} \Xi_{0}-2 q$.

REMARK 3.27. a) According to the remark 3.5a) for any point $(x, p)$ belonging to a $q-\pi$-submanifold $M$ of $T X$ then there exists a connected open neighborhood $U$ which is a $q-\pi$-submanifold of $T X$. If $M$ is a completely reducible $q-\pi$-submanifold of $T X$ then $U$ is a completely reducible $q-\pi$-submanifold of $T X$, and $C(U)$ the core of $U$ is included in the core of $M$. Obviously, for any point $(x, p)$ of $C(U)$ the index of $(x, p)$ seen as a point of $C(U)$ is equal to the index of $(x, p)$ seen as a point of $C(M)$. Generally speaking, any open set $U$ of a $q-\pi$-submanifold $M$ of $T X$ is a $q-\pi$-submanifold of $T X$ and if $M$ is a completely reducible $q-\pi$-submanifold then $U$ is a completely reducible $q-\pi$-submanifold. Thus, for any point $(x, p)$ of $M$ such that there exists an open set $U$ which is a completely reducible $q-\pi$-submanifold such that $(x, p)$ belongs to $C(U)$, then the index of $(x, p)$ seen as a point of $C(U)$ does not depend on $U$; when $M$ is a completely reducible $q-\pi$-submanifold, it is equal to the index of $(x, p)$ see as a point of $C(M)$.

b) As in the remark $3.5 \mathrm{~b}$ ), when $M$ is not a completely reducible $q-\pi$ submanifold of $T X$ we can consider the set, possibly empty, of points $(x, p)$ of $M$ such that there exists an open set $U$ of $M$ which is a completely reducible $q$ - $\pi$-submanifold of $T X$. Then, this set is an open set in $M$ and it is a completely reducible $q-\pi$-submanifold of $T X$. In the example 3.20 , it is the set of points $(x, p)$ of $T \mathbb{R}^{3}$ such that $p_{3}$ is not equal to zero.

REMARK 3.28. According to the remark 3.19 for a completely reducible $q-$ $\pi$-submanifold $M$ of class $C^{l}, l \geq m+2-2 q$, the $q-\pi$-submanifolds $M_{k}$ of the chain of reduction are of class $C^{l-k}$. Clearly, the core $C(M)$, which is the $q-\pi$-submanifold $M_{m+1-2 q}$ is a $q-\pi$-submanifold of class $C^{l-m-1+2 q}$. When $M$ has class $C^{l}$ with $l<m+2-2 q$ then, the chain of reduction is only defined for $k \leq l$ since the reduction $M_{l}$ is not defined. Consequently, if $M_{l}$ the reduction of $M_{l-1}$ is not equal to $M_{l}$, it is not possible to construct $C(M)$ the core of $M$. Obviously, when $M_{k+1}$ the reduction of $M_{k}$ is equal to $M_{k}$ with $k \leq l-1$ then, the core $C(M)$ is equal to $M_{k}$. 
EXAMPLE 3.29. The controlled rigid pendulum. The connected submanifold $C(M)$ has an index equal to 2 .

ExAMPLE 3.30. Under the mild assumption of the example 3.8 the connected manifold $M=\chi(X \times U)$ has an index equal to 0 .

According to the above theorem the core $C(M)$ of a completely reducible $q-\pi$-submanifold $M$ is the completely reducible $q-\pi$-submanifold $M_{m+1-2 q}$ which is reducible and equal to its reduction. Thus we can formulate the following theorem

Theorem 3.31. (Existence and uniqueness) Given $X$ a manifold and $M a$ completely reducible $q-\pi$-submanifold then its core $C(M)$ is either empty or a reducible $q-\pi$-submanifold equal to its reduction and for any point $(x, p)$ of $M$ there exists a local projection $W=\pi(V)$ of $M$ at $(x, p)$, an open set $O$ of $\mathbb{R}^{q}$ and a unique smooth mapping $\chi: W \times O \rightarrow T W$ such that

$$
V=\chi(W \times O), p=\chi(x, 0), \operatorname{rank} \frac{\partial \chi}{\partial u}(x, 0)=q
$$

and such that $\operatorname{Pr}=\pi \circ \chi$ where $P r$ is the canonical projection from $W \times O$ onto $W$. Moreover, if $W^{\prime}=\pi\left(V^{\prime}\right)$ is another local projection of $M$ at $(x, p)$ such that there exists an open set $O^{\prime}$ of $\mathbb{R}^{q}$ and a unique smooth mapping $\chi^{\prime}: W^{\prime} \times O^{\prime} \rightarrow T W^{\prime}$ such that

$$
V^{\prime}=\chi\left(W^{\prime} \times O^{\prime}\right), p=\chi^{\prime}(x, 0), \operatorname{rank} \frac{\partial \chi^{\prime}}{\partial u^{\prime}}(x, 0)=q
$$

and such that $P r^{\prime}=\pi \circ \chi^{\prime}$ where $P r^{\prime}$ is the canonical projection from $W^{\prime} \times O^{\prime}$ onto $W^{\prime}$, then there exists a diffeomorphism $h:(W \times O,(x, 0)) \rightarrow\left(W^{\prime} \times\right.$ $\left.O^{\prime},(x, 0)\right)$ such that $\chi=\chi^{\prime} \circ h$ and $\operatorname{Pr}=P r^{\prime} \circ h$.

REMARK 3.32. In our definition of a $q$ - $\pi$-submanifold we assume that the integer $q$ is the same for each connected component $\Xi$ of $M$; in fact we can extend the definition if we assume that the integer $q$ depends on the connected component $\Xi$. In other words $M$, is a disjoint reunion of $q_{i}-\pi$ submanifold $N_{i}$ of $T X$ of dimension $n_{i}, i \geq 1$, where the $q_{i}$ are integers less than or equal to $\operatorname{dim} X$. We will say again that $M$ is a $q-\pi$-submanifold, where $q$ is an integer $n$-tuple $\left(q_{1}, q_{2}, \ldots, q_{n}\right)$ ( $n$ possibly infinite). On the other hand the definition of the reduction is still valid and $M$ is a (completely) reducible $q-\pi$-submanifold of $T X$ if each $q_{i}-\pi$-submanifold $N_{i}$ is a (completely) reducible $q_{i}-\pi$-submanifold of $T X$. For a completely reducible $q-\pi$-submanifold $M$ of $T X$ we can define in the same way the chain of reduction $\left\{M_{k}\right\}_{k \geq 0}$. Clearly, if for each $q_{i}-\pi$-submanifold $N_{i},\left\{N_{i k}\right\}_{k \geq 0}$ is its chain of reduction, then, $M_{k}=\bigcup_{i} N_{i k}$. Moreover, according to the theorem 3.25 for each $q_{i}$ - $\pi$-submanifold $N_{i}$ of $T X$, the reduction $N_{n_{i}+2-2 q_{i}}$ of $N_{n_{i}+1-2 q_{i}}$ is $N_{n_{i}+1-2 q_{i}}$; consequently, if we pose $\alpha=\max \left\{n_{i}+2-2 q, i \geq 1\right\}$ then $M_{\alpha}$ the reduction of $M_{\alpha-1}$ is exactly $M_{\alpha-1}$. Then, the core is the $q-\pi$-submanifold $M_{\alpha-1}$. Clearly, the theorem 3.31 still holds.

\section{Algorithm OF REDUCTION}

For a $q-\pi$-submanifold $M$ we can set the following algorithm in a neighborhoods of any point $\left(x_{0}, p_{0}\right)$ of $M$, allowing us to know if $M$ is completely reducible, to find $C(M)$ and to obtain the controlled vector field. 
Step 0: Assume $M_{0}$ is a non-empty $q-\pi$-submanifold and $\left(x_{0}, p_{0}\right)$ belongs to $M_{0}=M$. Let $\Xi_{0}$ be the connected component of $M_{0}$ which contains $\left(x_{0}, p_{0}\right)$. From the definition of a $q-\pi$-submanifold there exists an open set $U_{0}$ of $\left(x_{0}, p_{0}\right)$ in $\Xi_{0}$, a submanifold $Y_{0}$ of $X$ such that $\operatorname{dim} Y_{0}+q=m_{0}+q=\operatorname{dim} \Xi_{0}$ and we have also seen that $U_{0}$ is a submanifold of $T Y_{0}$. We can place ourselves in a chart of $Y_{0}$ at $x_{0}$, even if this means shrinking $U_{0}$. Thus $Y_{0}$ is an open set of $\mathbb{R}^{m_{0}}$, that we denote again by $Y_{0}, T Y_{0}=Y_{0} \times \mathbb{R}^{m_{0}}$ and $U_{0}$ is a submanifold of $Y_{0} \times \mathbb{R}^{m_{0}}$ of dimension $m_{0}+q$. Then there exists in a neighborhood of $\left(x_{0}, p_{0}\right)$ in $Y_{0} \times \mathbb{R}^{m_{0}}$ a submersion $G_{0}:\left(Y_{0} \times \mathbb{R}^{m_{0}},\left(x_{0}, p_{0}\right)\right) \rightarrow$ $\left(\mathbb{R}^{m_{0}-q}, 0\right)$ such that $U_{0}=G_{0}^{-1}(0)$. In this way $\pi_{\mid \Xi_{0}}$ is a subimmersion in a neighborhood of $\left(x_{0}, p_{0}\right)$ if, and only if, $D_{p} G_{0}(x, p)$ has constant rank $\rho_{0}=r_{0}-q \leq m_{0}-q$ in a neighborhood of $\left(x_{0}, p_{0}\right)$ in $U_{0}$. Then any local projection $W_{0}=\pi\left(V_{0}\right)$ of $U_{0}$ (or $\left.M_{0}\right)$ in $\left(x_{0}, p_{0}\right)$ is a submanifold of $Y_{0}$ of dimension $r_{0}$. Thus there exists in a neighborhood of $x_{0}$ in $Y_{0}$ a submersion $g_{0}:\left(Y_{0}, x_{0}\right) \rightarrow\left(\mathbb{R}^{m_{0}-r_{0}}, 0\right)$ such that $W_{0}=g_{0}^{-1}(0)$ in a neighborhood of $x_{0}$ in $Y_{0}$. The tangent bundle $T W_{0}$ is the subset of points $(x, p)$ of $T Y_{0}$ for which the following equations are satisfied

$$
g_{0}(x)=0, \quad D g_{0}(x) p=0 .
$$

According to the definition 3.12 the reduction $M_{1}$ of $M_{0}$ is in a neighborhood of $\left(x_{0}, p_{0}\right)$ the (possibly empty) subset of points $(x, p)$ such that

$$
D g_{0}(x) p=0, \quad G_{0}(x, p)=0 .
$$

More particularly here, $\left(x_{0}, p_{0}\right)$ is a point of reducibility of $M_{0}$ if, and only if, $D g_{0}\left(x_{0}\right) p_{0}=0$. If $M_{1}$ is not a $q-\pi$-submanifold of $T X$ then the algorithm is stopped. If $M_{1}$ is empty then $C(M)$ is empty.

Step $k$ : Assume $M_{k}$ is a a non-empty $q-\pi$-submanifold and $\left(x_{0}, p_{0}\right)$ belongs to $M_{k}$. Let $\Xi_{k}$ be the connected component of $M_{k}$ which contains $\left(x_{0}, p_{0}\right)$. As before there exists an open set $U_{k}$ of $\left(x_{0}, p_{0}\right)$ in $\Xi_{k}$, a submanifold $Y_{k}$ of $X$ such that $\operatorname{dim} Y_{k}+q=m_{k}+q=\operatorname{dim} \Xi_{k}$ and $U_{k}$ is a submanifold of $T Y_{k}$. Even if this means shrinking $U_{k}$, let us place ourselves once more in a chart of $Y_{k}$ at $x_{0}$. Then $Y_{k}$ is an open set of $\mathbb{R}^{m_{k}}$ that we denote by $Y_{k}$, $T Y_{k}=Y_{k} \times \mathbb{R}^{m_{k}}$ and $U_{k}$ is a submanifold of $Y_{k} \times \mathbb{R}^{m_{k}}$ of dimension $m_{k}+q$. Then there exists in a neighborhood of $\left(x_{0}, p_{0}\right)$ in $Y_{k} \times \mathbb{R}^{m_{k}}$ a submersion $G_{k}$ : $\left(Y_{k} \times \mathbb{R}^{m_{k}},\left(x_{0}, p_{0}\right)\right) \rightarrow\left(\mathbb{R}^{m_{k}-q}, 0\right)$ such that $U_{k}=G_{k}^{-1}(0)$. In this way $\pi_{\mid \Xi_{k}}$ is a subimmersion in a neighborhood of $\left(x_{0}, p_{0}\right)$, if, and only if, $D_{p} G_{k}(x, p)$ has constant rank $\rho_{k}=r_{k}-q \leq m_{k}-q$ in a neighborhood of $\left(x_{0}, p_{0}\right)$ in $U_{k}$. Then any local projection $W_{k}=\pi\left(V_{k}\right)$ of $U_{k}$ (or $\left.M_{k}\right)$ in $\left(x_{0}, p_{0}\right)$ is a submanifold of $Y_{k}$ of dimension $r_{k}$. There exists in a neighborhood of $x_{0}$ in $Y_{k}$ a submersion $g_{k}:\left(Y_{0}, x_{0}\right) \rightarrow\left(\mathbb{R}^{m_{k}-r_{k}}, 0\right)$ such that $W_{k}=g_{k}^{-1}(0)$ in a neighborhood of $x_{0}$ in $Y_{k}$. The tangent bundle $T W_{k}$ is the subset of points $(x, p)$ of $T Y_{k}$ for which the following equations are satisfied

$$
g_{k}(x)=0, \quad D g_{k}(x) p=0 .
$$

The reduction $M_{k+1}$ of $M_{k}$ is in a neighborhood of $\left(x_{0}, p_{0}\right)$ the (possibly empty) subset of points such that

$$
D g_{k}(x) p=0, \quad G_{k}(x, p)=0 .
$$


More particularly here, $\left(x_{0}, p_{0}\right)$ is a point of reducibility of $M_{k}$ if, and only if, $D g_{k}\left(x_{0}\right) p_{0}=0$. If $M_{k+1}$ is not a $q$ - $\pi$-submanifold then the algorithm is stopped. If $M_{k+1}$ is empty then $C(M)$ is empty.

Step $m+1-2 q$ : Let us assume that $M_{m+1-2 q}$ is a non-empty $q-\pi$ submanifold of $T X$ and $\left(x_{0}, p_{0}\right)$ belongs to $M_{m+1-2 q}$. Then the proof of the theorem 3.10 gives the controlled vector field.

REMARK 4.1. For each step $k$ we define the submersions

$$
\begin{gathered}
G_{k}:\left(Y_{k} \times \mathbb{R}^{m_{k}},\left(x_{0}, p_{0}\right)\right) \rightarrow\left(\mathbb{R}^{m_{k}-q}, 0\right) \\
g_{k}:\left(Y_{k}, x_{0}\right) \rightarrow\left(\mathbb{R}^{m_{k}-r_{k}}, 0\right)
\end{gathered}
$$

with $m_{0}=\operatorname{dim} \Xi_{0}$ and $m_{k+1}=r_{k}=\rho_{k}+q \leq m_{k}$. Since the sequences of integers $\rho_{k}$ and $m_{k}$ are decreasing there exists an integer $\alpha$ such that $\rho_{k-1}=\rho_{\alpha-1}$ and $m_{k}=m_{\alpha}$ for any integer $k \geq \alpha$. We have the following sequence of inequalities

$$
q \leq \cdots=m_{\alpha+1}=m_{\alpha}<m_{\alpha-1}<\cdots<m_{k+1}<m_{k}<\cdots<m_{1}<m_{0}<n .
$$

We find once again that the index $\alpha$ is, at most, equal to $m_{0}-q=\operatorname{dim} \Xi_{0}-2 q$ and it is equal to $m_{0}-q$ when $m_{k+1}=m_{k}-1$ for all $k<\alpha$.

We shall end this section by showing first how to obtain $g_{k}$ from $G_{k}$, then how to characterize the reducibility of $M_{k}$ in a neighborhood of $\left(x_{0}, p_{0}\right)$ and finally how to find $G_{k+1}$ with $g_{k}$ and $G_{k}$.

Construction of $g_{k}$ : we show the existence of a local coordinates system on a submanifold $N_{k}$ of $U_{k}$ such that $\pi_{\mid N_{k}}: N_{k} \rightarrow W_{k}$ is a local diffeomorphism. Since, $N_{k}$ has to be a submanifold of $U_{k}$ of dimension $r_{k}$, we must obtain a submersion $\tilde{G}_{k}$ of $Y_{k} \times \mathbb{R}^{m_{k}}$ in $\mathbb{R}^{2 m_{k}-r_{k}}$. Let $A_{k}: \mathbb{R}^{m_{k}} \rightarrow \mathbb{R}^{m_{k}-\rho_{k}}$ be a linear mapping such that

$$
\text { ker } A_{k} \cap \operatorname{ker} D_{p} G_{k}\left(x_{0}, p_{0}\right)=\{0\}, \quad \operatorname{rank} A_{k}=m_{k}-\rho_{k}
$$

We define $\tilde{G}_{k}$ by $\tilde{G}_{k}(x, p)=\left(G_{k}(x, p), A_{k}\left(p-p_{0}\right)\right)$. D $\tilde{G}_{k}\left(x_{0}, p_{0}\right)$ has full rank $2 m_{k}-r_{k}$, and so $\tilde{G}_{k}$ is a submersion in an open neighborhood of $\left(x_{0}, p_{0}\right)$ in $T Y_{k}$. We take $N_{k}=\tilde{G}_{k}^{-1}(0)$ in a neighborhood of $\left(x_{0}, p_{0}\right)$ in $U_{k}$. The tangent space is

$$
T_{\left(x_{0}, p_{0}\right)} N_{k}=\operatorname{ker} D \tilde{G}_{k}\left(x_{0}, p_{0}\right)=\operatorname{ker} A_{k} \cap \operatorname{ker} D G_{k}\left(x_{0}, p_{0}\right) .
$$

Then, according to the following equality

$$
\begin{aligned}
& T_{\left(x_{0}, p_{0}\right)} N_{k} \cap \operatorname{ker} D\left(\pi_{\mid U_{k}}\right)\left(x_{0}, p_{0}\right)= \\
& \quad \operatorname{ker} D \tilde{G}_{k}\left(x_{0}, p_{0}\right) \cap\{0\} \times \operatorname{ker} D_{p} G_{k}\left(x_{0}, p_{0}\right)=0
\end{aligned}
$$

and the subimmersion theorem, $\pi_{\mid N_{k}}: N_{k} \rightarrow W_{k}$ is a local diffeomorphism.

The local coordinate system of $N_{k}$ in a neighborhood of $\left(x_{0}, p_{0}\right)$ is obtained in the following way. Let $E_{k}=\operatorname{Im} D_{p} G_{k}\left(x_{0}, p_{0}\right)$ and $F_{k}$ be any complement of $E_{k}$ in $\mathbb{R}^{m_{k}-q}$ and $P_{k}$ the projection of $\mathbb{R}^{m_{k}-q}=E_{k} \oplus F_{k} \simeq E_{k} \times F_{k}$ onto $F_{k}$.

Lemma 4.2. The subspace $K_{k}=\operatorname{ker} P_{k} D_{x} G_{k}\left(x_{0}, p_{0}\right)$ of $\mathbb{R}^{m_{k}}$ has dimension $r_{k}$. Moreover, for any complement $L_{k}$ of $K_{k}$ in $\mathbb{R}^{m_{k}}$, the linear mapping

$$
I_{k}=P_{k} D_{x} G_{k}\left(x_{0}, p_{0}\right)_{\mid L_{k}}: L_{k} \rightarrow F_{k}
$$

is an isomorphism. 
Proof. For the subspace $K_{k}$ we have the first inequality

$$
\operatorname{dim} K_{k}=m_{k}-\operatorname{dim} \operatorname{Im} P_{k} D_{x} G_{k}\left(x_{0}, p_{0}\right) \geq m_{k}-\left(m_{k}-r_{k}\right)=r_{k}
$$

Let us show the other inequality. According to the definition of the projection $P_{k}$

$$
P_{k} D G\left(x_{0}, p_{0}\right)=P_{k} D_{x} G_{k}\left(x_{0}, p_{0}\right) .
$$

Consequently, for any $(\delta x, \delta p) \in T_{\left(x_{0}, p_{0}\right)} N_{k}, \delta x \in K_{k}$. Conversely, for any $\delta x \in K_{k}$ there exists a $\delta p \in \mathbb{R}^{m_{k}}$ such that $(\delta x, \delta p) \in T_{\left(x_{0}, p_{0}\right)} N_{k}$. Indeed, if $\delta x \in K_{k}$, then $D_{x} G_{k}\left(x_{0}, p_{0}\right) \delta x \in \operatorname{Im} D_{p} G_{k}\left(x_{0}, p_{0}\right)$, and so there exists $\delta p^{\prime} \in$ $\mathbb{R}^{m_{k}}$ such that $D G_{k}\left(x_{0}, p_{0}\right)\left(\delta x, \delta p^{\prime}\right)=0$. But, since $A_{k}$ is an isomorphism from ker $D_{p} G_{k}\left(x_{0}, p_{0}\right)$ into $\mathbb{R}^{m_{k}-\rho_{k}}$ there exists a $\delta p^{\prime \prime} \in \operatorname{ker} D_{p} G_{k}\left(x_{0}, p_{0}\right)$ such that

$$
A_{k}\left(\delta p^{\prime}+\delta p^{\prime \prime}\right)=0
$$

whence $(\delta x, \delta p) \in T_{\left(x_{0}, p_{0}\right)} N_{k}$ for $\delta p=\delta p^{\prime}+\delta p^{\prime \prime}$. Thus

$$
D \pi\left(x_{0}, p_{0}\right) T_{\left(x_{0}, p_{0}\right)} N_{k}=K_{k}
$$

and $\operatorname{dim} K_{k} \leq \operatorname{dim} T_{\left(x_{0}, p_{0}\right)} N_{k}=r_{k}$. Finally, according to the definition of $L_{k}$ and $I_{k}$, ker $I_{k}=L_{k} \cap K_{k}=\{0\}$.

Thus any point $x$ of $\mathbb{R}^{m_{k}}$ is splitting in a unique way as $x=\bar{x}+\tilde{x}$, where $\bar{x}$ belongs to $K_{k}$ and $\tilde{x}$ belongs to $L_{k}$. Let us consider in a open neighborhood of $\left(x_{0}, p_{0}\right)$ the equation

$$
\tilde{H}_{k}(\bar{x}, \tilde{x}, p)=\tilde{G}_{k}(\bar{x}+\tilde{x}, p)=\left(G_{k}(\bar{x}+\tilde{x}, p), A_{k}\left(p-p_{0}\right)\right)=0 .
$$

Since

$$
D_{(\tilde{x}, p)} \tilde{H}\left(x_{0}, p_{0}\right)=\left(\begin{array}{cc}
\left.D_{x} G_{k}\left(x_{0}, p_{0}\right)\right|_{L_{k}} & D_{p} G\left(x_{0}, p_{0}\right) \\
0 & A\left(p-p_{0}\right)
\end{array}\right)
$$

is an isomorphism then in an open neighborhood of $\left(x_{0}, p_{0}\right)$

$$
N_{k}=\left\{\left(\bar{x}, \phi_{k}(\bar{x}), \psi_{k}(\bar{x})\right\}\right.
$$

with $\left(\bar{x}_{0}, \phi_{k}\left(\bar{x}_{0}\right), \psi_{k}\left(x_{0}\right)\right)=\left(\bar{x}_{0}, \tilde{x}_{0}, p_{0}\right)$. Lastly, in a neighborhood of $x_{0}$,

$$
W_{k}=\pi\left(N_{k}\right)=\left\{\left(\bar{x}, \phi_{k}(\bar{x})\right\}=g_{k}^{-1}(0) .\right.
$$

We can therefore establish $g_{k}(x)=\tilde{x}-\phi_{k}(\bar{x})$.

Criterion of reducibility: in order to characterize the reducibility of $M_{k}$ in a neighborhood of $\left(x_{0}, p_{0}\right)$ we give the following result

Proposition 4.3. Given $g_{k}: \mathbb{R}^{m_{k}}=K_{k} \oplus L_{k} \rightarrow L_{k} \simeq \mathbb{R}^{m_{k}-r_{k}}$ the above mapping, then for any $\delta x=\delta \bar{x}+\delta \tilde{x}, \delta y=\delta \bar{y}+\delta \tilde{y}$ of $\mathbb{R}^{m_{k}}$

$$
D g\left(x_{0}\right) \delta x=\delta \tilde{x} \text { and } D^{2} g_{k}\left(x_{0}\right)(\delta x, \delta y)=I_{k}^{-1} P_{k} B_{k}(\delta \bar{x}, \delta \bar{y})
$$

where $B_{k}$ is the bilinear mapping on $K_{k}$

$$
B_{k}(\bullet, \bullet)=D^{2} G_{k}\left(x_{0}, p_{0}\right)\left(\left(\bullet, D \psi_{k}\left(\bar{x}_{0}\right) \bullet\right),\left(\mathbf{\bullet}, D \psi_{k}\left(\bar{x}_{0}\right) \bullet\right)\right)
$$

Proof. For any $\delta x=\delta \bar{x}+\delta \tilde{x}, \delta y=\delta \bar{y}+\delta \tilde{y}$ of $\mathbb{R}^{m_{k}}$

$$
\begin{gathered}
D g_{k}\left(x_{0}\right) \delta x=\delta \tilde{x}-D \phi_{k}\left(\bar{x}_{0}\right) \delta \bar{x} \\
D^{2} g_{k}\left(x_{0}\right)(\delta x, \delta y)=-D^{2} \phi_{k}\left(\bar{x}_{0}\right)(\delta \bar{x}, \delta \bar{y}) .
\end{gathered}
$$

According to the relationship $\tilde{H}_{k}\left(\bar{x}, \phi_{k}(\bar{x}), \psi_{k}(\bar{x})\right)=0$ we have

$$
D_{\bar{x}} \tilde{H}_{k}\left(\bar{x}, \phi_{k}(\bar{x}), \psi_{k}(\bar{x})\right)+D_{\tilde{x}} \tilde{H}_{k}\left(\bar{x}, \phi_{k}(\bar{x}), \psi_{k}(\bar{x})\right) D \phi_{k}(\bar{x})
$$




$$
+D_{p} \tilde{H}_{k}\left(\bar{x}, \phi_{k}(\bar{x}), \psi_{k}(\bar{x})\right) D \psi_{k}(\bar{x})=0,
$$

and at the point $\left(x_{0}, p_{0}\right)$ this gives

$$
\begin{gathered}
D_{\bar{x}} \tilde{H}_{k}\left(\bar{x}_{0}, \tilde{x}_{0}, p_{0}\right)+D_{\tilde{x}} \tilde{H}_{k}\left(\bar{x}_{0}, \tilde{x}_{0}, p_{0}\right) D \phi_{k}\left(\bar{x}_{0}\right) \\
+D_{p} \tilde{H}_{k}\left(\bar{x}_{0}, \tilde{x}_{0}, p_{0}\right) D \psi_{k}\left(\bar{x}_{0}\right)=0 .
\end{gathered}
$$

But

$$
\begin{aligned}
D_{\bar{x}} \tilde{H}_{k}\left(\bar{x}_{0}, \tilde{x}_{0}, p_{0}\right) & =\left(\begin{array}{c}
D_{x} G_{k}\left(x_{0}, p_{0}\right)_{\mid K_{k}} \\
0
\end{array}\right) \\
D_{\tilde{x}} \tilde{H}_{k}\left(\bar{x}_{0}, \tilde{x}_{0}, p_{0}\right) & =\left(\begin{array}{c}
D_{x} G_{k}\left(x_{0}, p_{0}\right)_{\mid L_{k}} \\
0
\end{array}\right) \\
D_{p} \tilde{H}_{k}\left(\bar{x}_{0}, \tilde{x}_{0}, p_{0}\right) & =\left(\begin{array}{c}
D_{p} G_{k}\left(x_{0}, p_{0}\right) \\
A_{k}
\end{array}\right) .
\end{aligned}
$$

Thus, we obtain

$$
\begin{gathered}
D_{x} G_{k}\left(x_{0}, p_{0}\right)_{\mid K_{k}}+D_{x} G_{k}\left(x_{0}, p_{0}\right)_{\mid L_{k}} D \phi_{k}\left(\bar{x}_{0}\right)+D_{p} G_{k}\left(x_{0}, p_{0}\right) D \psi_{k}\left(\bar{x}_{0}\right)=0 \\
A_{k} D \psi_{k}\left(\bar{x}_{0}\right)=0 .
\end{gathered}
$$

Let us apply $P_{k}$ on the second equality, then we find $D \phi_{k}\left(\bar{x}_{0}\right)=0$.

According to the relationship

$$
\begin{aligned}
& D_{\bar{x}} \tilde{H}_{k}\left(\bar{x}, \phi_{k}(\bar{x}), \psi_{k}(\bar{x})\right)+D_{\tilde{x}} \tilde{H}_{k}\left(\bar{x}, \phi_{k}(\bar{x}), \psi_{k}(\bar{x})\right) D \phi_{k}(\bar{x}) \\
& \quad+D_{p} \tilde{H}_{k}\left(\bar{x}, \phi_{k}(\bar{x}), \psi_{k}(\bar{x})\right) D \psi_{k}(\bar{x})=0,
\end{aligned}
$$

we obtain at the point $(\bar{x}, \tilde{x}, p)=\left(\bar{x}, \phi_{k}(\bar{x}), \psi_{k}(\bar{x})\right.$ the following equality

$$
\begin{array}{ll}
D_{\bar{x}^{2}}^{2} \tilde{H}_{k}(\bar{x}, \tilde{x}, p)(\delta \bar{x}, \delta \bar{y})+D_{\tilde{x} \bar{x}}^{2} \tilde{H}_{k}(\bar{x}, \tilde{x}, p)\left(\delta \bar{x}, D \phi_{k}(\bar{x}) \delta \bar{y}\right) \\
\quad+\quad D_{p \bar{x}}^{2} \tilde{H}_{k}(\bar{x}, \tilde{x}, p)\left(\delta \bar{x}, D \psi_{k}(\bar{x}) \delta \bar{y}\right)+D_{\bar{x} \tilde{x}}^{2} \tilde{H}_{k}(\bar{x}, \tilde{x}, p)\left(D \phi_{k}(\bar{x}) \delta \bar{x}, \delta \bar{y}\right) \\
+\quad D_{\tilde{x}^{2}}^{2} \tilde{H}_{k}(\bar{x}, \tilde{x}, p)\left(D \phi_{k}(\bar{x}) \delta \bar{x}, D \phi_{k}(\bar{x}) \delta \bar{y}\right) \\
+\quad D_{p \tilde{x}}^{2} \tilde{H}_{k}(\bar{x}, \tilde{x}, p)\left(D \phi_{k}(\bar{x}) \delta \bar{x}, D \psi_{k}(\bar{x}) \delta \bar{y}\right) \\
+\quad D_{\tilde{x}} \tilde{H}_{k}(\bar{x}, \tilde{x}, p) D^{2} \phi_{k}(\bar{x})(\delta \bar{x}, \delta \bar{y})+D_{\bar{x} p}^{2} \tilde{H}_{k}(\bar{x}, \tilde{x}, p)\left(D \psi_{k}(\bar{x}) \delta \bar{x}, \delta \bar{y}\right) \\
+\quad D_{\tilde{x} p}^{2} \tilde{H}_{k}(\bar{x}, \tilde{x}, p)\left(D \psi_{k}(\bar{x}) \delta \bar{x}, D \phi_{k}(\bar{x}) \delta \bar{y}\right) \\
+\quad D_{p^{2}}^{2} \tilde{H}_{k}(\bar{x}, \tilde{x}, p)\left(D \psi_{k}(\bar{x}) \delta \bar{x}, D \psi_{k}(\bar{x}) \delta \bar{y}\right) \\
+\quad D_{p} \tilde{H}_{k}(\bar{x}, \tilde{x}, p) D^{2} \psi_{k}(\bar{x})(\delta \bar{x}, \delta \bar{y})=0
\end{array}
$$

which thus gives at the point $\left(x_{0}, p_{0}\right)$ the equality

$$
\begin{aligned}
& D_{\bar{x}^{2}}^{2} \tilde{H}_{k}\left(\bar{x}_{0}, \tilde{x}_{0}, p_{0}\right)(\delta \bar{x}, \delta \bar{y})+D_{\bar{x} p}^{2} \tilde{H}_{k}\left(\bar{x}_{0}, \tilde{x}_{0}, p_{0}\right)\left(D \psi_{k}\left(\bar{x}_{0}\right) \delta \bar{x}, \delta \bar{y}\right) \\
& \quad+\quad D_{p \bar{x}}^{2} \tilde{H}_{k}\left(\bar{x}_{0}, \tilde{x}_{0}, p_{0}\right)\left(\delta \bar{x}, D \psi_{k}\left(\bar{x}_{0}\right) \delta \bar{y}\right) \\
& \quad+\quad D_{p^{2}}^{2} \tilde{H}_{k}\left(\bar{x}_{0}, \tilde{x}_{0}, p_{0}\right)\left(D \psi_{k}\left(\bar{x}_{0}\right) \delta \bar{x}, D \psi_{k}\left(\bar{x}_{0}\right) \delta \bar{y}\right) \\
& \quad+\quad D_{p} \tilde{H}_{k}\left(\bar{x}_{0}, \tilde{x}_{0}, p_{0}\right) D^{2} \psi_{k}\left(\bar{x}_{0}\right)(\delta \bar{x}, \delta \bar{y}) \\
& \quad+\quad D_{\tilde{x}} \tilde{H}_{k}\left(\bar{x}_{0}, \tilde{x}_{0}, p_{0}\right) D^{2} \phi_{k}\left(\bar{x}_{0}\right)(\delta \bar{x}, \delta \bar{y})=0 .
\end{aligned}
$$

According to the definition of $\tilde{H}_{k}$ we obtain for $G_{k}$ the relationship

$$
\begin{aligned}
& D_{x} G_{k}\left(x_{0}, p_{0}\right)_{\mid L_{k}} D^{2} \phi_{k}\left(\bar{x}_{0}\right)(\delta \bar{x}, \delta \bar{y})= \\
& \quad-\quad D_{p} G_{k}\left(x_{0}, p_{0}\right) D^{2} \psi_{k}\left(\bar{x}_{0}\right)(\delta \bar{x}, \delta \bar{y}) \\
& -\quad D^{2} G_{k}\left(x_{0}, p_{0}\right)\left(\left(\delta \bar{x}, D \psi_{k}\left(\bar{x}_{0}\right) \delta \bar{x}\right),\left(\delta \bar{y}, D \psi_{k}\left(\bar{x}_{0}\right) \delta \bar{y}\right)\right) .
\end{aligned}
$$


Let us apply $P_{k}$ on the left, then we obtain

$$
\begin{aligned}
& I_{k} D^{2} \phi_{k}\left(\bar{x}_{0}\right)(\delta \bar{x}, \delta \bar{y})= \\
& \quad-P_{k} D^{2} G_{k}\left(x_{0}, p_{0}\right)\left(\left(\delta \bar{x}, D \psi_{k}\left(\bar{x}_{0}\right) \delta \bar{x}\right),\left(\delta \bar{y}, D \psi_{k}\left(\bar{x}_{0}\right) \delta \bar{y}\right)\right) .
\end{aligned}
$$

Proposition 4.4. Let $\left(x_{0}, p_{0}\right)$ be a point of reducibility of $M_{k}$ and $W_{k}=$ $\pi(V)$ a local projection of $M_{k}$ in $\left(x_{0}, p_{0}\right) . M_{k}$ is reducible in a neighborhood of $\left(x_{0}, p_{0}\right)$ if, and only if,

$$
\operatorname{rank}\left(\begin{array}{cc}
P_{k} B_{k}\left(p_{0}, \bullet\right) & P_{k} D_{x} G_{k}\left(x_{0}, p_{0}\right) \\
D_{x} G_{k}\left(x_{0}, p_{0}\right) & D_{p} G_{k}\left(x_{0}, p_{0}\right)
\end{array}\right)=2 m_{k}-\left(r_{k}+q\right)
$$

and if in a neighborhood of $\left(x_{0}, p_{0}\right)$ in $T W_{k} \cap V_{k}$

$$
\begin{array}{r}
\operatorname{dim}\left[\operatorname{ker} P_{k}(x, p) D_{x} G_{k}(x, p) \cap \operatorname{ker} D_{p} G_{k}(x, p)\right]= \\
\quad \operatorname{dim}\left[\operatorname{ker} P_{k} D_{x} G_{k}\left(x_{0}, p_{0}\right) \cap \operatorname{ker} D_{p} G_{k}\left(x_{0}, p_{0}\right)\right]
\end{array}
$$

where $P_{k}(x, p)$ is the projection onto a complement of

$$
E_{k}(x, p)=\operatorname{Im} D_{p} G_{k}(x, p) .
$$

Proof. Clearly, the tangent space of $T W_{k}$ at $\left(x_{0}, p_{0}\right)$ is the subset of points $(\delta x, \delta p)$ of $T Y_{k}$ which satisfy the following system

$$
\begin{gathered}
D g_{k}\left(x_{0}\right) \delta x=0 \\
D^{2} g_{k}\left(x_{0}\right)\left(p_{0}, \delta x\right)+D g_{k}\left(x_{0}\right) \delta p=0
\end{gathered}
$$

and consequently $T_{\left(x_{0}, p_{0}\right)} T W_{k} \cap T_{\left(x_{0}, p_{0}\right)} M_{k}$ is the subset of points such that

$$
\begin{gathered}
D g_{k}\left(x_{0}\right) \delta x=0 \\
D^{2} g_{k}\left(x_{0}\right)\left(p_{0}, \delta x\right)+D g_{k}\left(x_{0}\right) \delta p=0 \\
D_{x} G_{k}\left(x_{0}, p_{0}\right) \delta x+D_{p} G_{k}\left(x_{0}, p_{0}\right) \delta p=0 .
\end{gathered}
$$

But, on the one hand, according to proposition 4.3, the first equality means that $\delta x=\delta \bar{x}$, namely $\delta x$ belongs to $K_{k}$. And on the other hand, we obtain the same thing when $P_{k}$ is applied on the third equation. Thus we can leave aside the first equation. Now, $T_{\left(x_{0}, p_{0}\right)} T W_{k} \cap T_{\left(x_{0}, p_{0}\right)} M_{k}$ is the kernel of the linear mapping $D R_{k}\left(x_{0}, p_{0}\right): \mathbb{R}^{m_{k}} \times \mathbb{R}^{m_{k}} \rightarrow L_{k} \times \mathbb{R}^{m_{k}-q}$ where

$$
D R_{k}(x, p)=\left(\begin{array}{cc}
D^{2} g_{k}(x)(p, \bullet) & D g_{k}(x) \\
D_{x} G_{k}(x, p) & D_{p} G_{k}(x, p)
\end{array}\right) .
$$

Therefore, the subspace $T_{\left(x_{0}, p_{0}\right)} T W_{k} \cap T_{\left(x_{0}, p_{0}\right)} M$ has dimension $r_{k}+q$ if, and only if, the linear mapping $D R_{k}\left(x_{0}, p_{0}\right)$ has full rank $m_{k}-r_{k}+m_{k}-q=$ $2 m_{k}-\left(r_{k}+q\right)$, namely if, and only if, $\operatorname{dim} \operatorname{ker} D R_{k}\left(x_{0}, p_{0}\right)=r_{k}+q$. A point $(\delta x, \delta p)$ belongs to ker $D R_{k}\left(x_{0}, p_{0}\right)$ if, and only if, $(\delta x, \delta p)$ satisfy the following system

$$
\left\{\begin{array}{cc}
D^{2} g_{k}\left(x_{0}\right)\left(p_{0}, \delta x\right)+D g_{k}\left(x_{0}\right) \delta \tilde{p} & =0 \\
D_{x} G_{k}\left(x_{0}, p_{0}\right) \delta x+D_{p} G_{k}\left(x_{0}, p_{0}\right) \delta p & =0
\end{array}\right.
$$

According to proposition 4.3 this is equivalent to the system

$$
\left\{\begin{array}{cc}
I_{k}^{-1} P_{k} B_{k}\left(p_{0}, \delta \bar{x}\right)+\delta \tilde{p} & =0 \\
D_{x} G_{k}\left(x_{0}, p_{0}\right) \delta x+D_{p} G_{k}\left(x_{0}, p_{0}\right) \delta p & =0
\end{array}\right.
$$


And according to lemma 4.2 this is equivalent to the system

$$
\left\{\begin{array}{l}
P_{k} B_{k}\left(p_{0}, \delta \bar{x}\right)+P_{k} D_{x} G_{k}\left(x_{0}, p_{0}\right) \delta \tilde{p}=0 \\
D_{x} G_{k}\left(x_{0}, p_{0}\right) \delta x+D_{p} G_{k}\left(x_{0}, p_{0}\right) \delta p=0 .
\end{array}\right.
$$

Lastly, as we have seen above, the second equation implies that $\delta x=\delta \bar{x}$. Thus, we are able to write $\delta x$ instead of $\delta \bar{x}$ in the first equation of this system. Obviously we are also able to write $\delta p$ instead of $\delta \tilde{p}$ in the first equation. Then we obtain the system

$$
\left\{\begin{array}{l}
P_{k} B_{k}\left(p_{0}, \delta x\right)+P_{k} D_{x} G_{k}\left(x_{0}, p_{0}\right) \delta p=0 \\
D_{x} G_{k}\left(x_{0}, p_{0}\right) \delta x+D_{p} G_{k}\left(x_{0}, p_{0}\right) \delta p=0
\end{array}\right.
$$

Thus, dim ker $D R_{k}\left(x_{0}, p_{0}\right)=r_{k}+q_{k}$ if, and only if, (4.1) holds.

Now, assume that in an open neighborhood of $\left(x_{0}, p_{0}\right)$ in $T W_{k} \cap V_{k}$

$$
\text { rank } \pi_{\mid T_{(x, p)}\left(T W_{k} \cap V_{k}\right)} \text { is constant. }
$$

As we can see, $D R_{k}\left(x_{0}, p_{0}\right)$ is precisely the derivative of the mapping

$$
\begin{aligned}
R_{k} & : T Y_{k} \rightarrow L_{k} \times \mathbb{R}^{m_{k}-q} \\
(x, p) & \mapsto\left(D g_{k}(x, p), G_{k}(x, p)\right)
\end{aligned}
$$

where the zero set in a neighborhood of $\left(x_{0}, p_{0}\right)$ is exactly $T W_{k} \cap V_{k}$. Thus, in a neighborhood of $\left(x_{0}, p_{0}\right), T_{(x, p)}\left(T W_{k} \cap V_{k}\right)$ is the kernel of $D R_{k}(x, p)$. The condition (4.2) holds if, and only if, the mapping

$$
(\delta x, \delta p) \in \operatorname{ker} D R_{k}(x, p) \mapsto \delta x
$$

has constant rank in neighborhood of $\left(x_{0}, p_{0}\right)$ in $T W_{k} \cap V_{k}$. Compute the kernel of this mapping for any point $(x, p)$ in an open neighborhood of $\left(x_{0}, p_{0}\right)$. This is the set of $(\delta x, \delta p)$ of $\mathbb{R}^{m_{k}} \times \mathbb{R}^{m_{k}}$ such that

$$
\begin{gathered}
\delta x=0 \\
D^{2} g_{k}(x)(p, \delta x)+D g_{k}(x) \delta p=0 \\
D_{x} G_{k}(x, p) \delta x+D_{p} G_{k}(x, p) \delta p=0
\end{gathered}
$$

namely, the set $\{0\} \times\left[\operatorname{ker} D g_{k}(x) \cap \operatorname{ker} D_{p} G_{k}(x, p)\right]$. However,

$$
\text { ker } D g_{k}(x)=\operatorname{ker} P_{k}(x, p) D_{x} G_{k}(x, p) \text {. }
$$

Indeed the equality $D g_{k}(x) \delta p=0$ means that $\delta p$ belongs to $T_{x} W_{k}$ which is the projection of $T_{x} M_{k}$. Therefore, there exists $\delta q$ such that $D_{x} G_{k}(x, p) \delta p+$ $D_{p} G_{k}(x, p) \delta q=0$. And if we apply $P_{k}(x, p)$, we obtain that

$$
\text { ker } D g_{k}(x) \subset \operatorname{ker} P_{k}(x, p) D_{x} G_{k}(x, p) \text {. }
$$

Since $\operatorname{dim} \operatorname{ker} D g_{k}(x)=\operatorname{dim} \operatorname{ker} P_{k}(x, p) D_{x} G_{k}(x, p)$ we have the equality. Thus, the mapping (4.3) has constant rank if, and only if,

$$
\begin{array}{r}
\operatorname{dim}\left[\operatorname{ker} P_{k}(x, p) D_{x} G_{k}(x, p) \cap \operatorname{ker} D_{p} G_{k}(x, p)\right]= \\
\operatorname{dim}\left[\operatorname{ker} P_{k} D_{x} G_{k}\left(x_{0}, p_{0}\right) \cap \operatorname{ker} D_{p} G_{k}\left(x_{0}, p_{0}\right)\right]
\end{array}
$$

Construction of $G_{k+1}$ : we construct a nother submersion for $M_{k}$ and then we obtain a submersion for $M_{k+1}$. Let $C_{k}$ be a linear mapping from $\mathbb{R}^{m_{k}-\left(\rho_{k}+q\right)}$ to $\mathbb{R}^{m_{k}-q}$ such that $\operatorname{Im} C_{k} \oplus E_{k}=\mathbb{R}^{m_{k}-q 4}, \mathcal{K}_{k}$ the kernel of $D_{p} G_{k}\left(x_{0}, p_{0}\right)$ and $\mathcal{L}_{k}$ a complement of $\mathcal{K}_{k}$. The linear mapping $J_{k}: \mathcal{L}_{k} \times \mathbb{R}^{m_{k}-\left(\rho_{k}+q\right)} \rightarrow$

\footnotetext{
${ }^{4}$ Such mapping is one-to-one since it has rank $m_{k}-\left(\rho_{k}+q\right)$.
} 
$\mathbb{R}^{m_{k}-q}$ given by $J_{k}(u, v)=D_{p} G_{k}\left(x_{0}, p_{0}\right) u+C_{k} v$ is an isomorphism. Indeed, for any $(u, v)$ such that $D_{p} G_{k}\left(x_{0}, p_{0}\right) u+C_{k} v=0$ since $u$ belongs to $\mathcal{L}_{k}$ a complement of $\mathcal{K}_{k}=\operatorname{ker} D_{p} G_{k}\left(x_{0}, p_{0}\right)$, then $C_{k} v$ belongs to $\operatorname{Im} D_{p} G_{k}\left(x_{0}, p_{0}\right)=$ $E_{k}$, therefore $C_{k} v=0$, and $v=0$ since $C_{k}$ is one-to-one. Consequently, $u$ belongs to $\mathcal{K}_{k}$ and then $u=0$. Now, for the mapping

$$
\begin{gathered}
F_{k}: T Y_{k} \times \mathbb{R}^{m_{k}-\left(\rho_{k}+q\right)} \rightarrow \mathbb{R}^{m_{k}-q} \\
(x, p, u) \mapsto G_{k}(x, p)+C_{k} u
\end{gathered}
$$

the zero set $\tilde{M}_{k}$ is a submanifold of dimension $2 m_{k}-\rho_{k}$. Using the implicit mapping theorem we obtain the following parameterization of $\tilde{M}_{k}$

$$
\check{p}=a_{k}(x, \hat{p}), \quad u=b_{k}(x, \hat{p})
$$

where $\check{p}$ belongs to $\mathcal{L}_{k}$ and $\hat{p}$ to $\mathcal{K}_{k}$. Then we define in a neighborhood of $\left(x_{0}, p_{0}\right)$ in $T Y_{k}$ the mapping

$$
\begin{gathered}
\tilde{F}_{k}: T Y_{k} \rightarrow \mathcal{L}_{k} \simeq \mathbb{R}^{\rho_{k}} \\
(x, p) \mapsto \tilde{F}_{k}(x, p)=\check{p}-a_{k}(x, \hat{p})
\end{gathered}
$$

and the mapping

$$
\begin{gathered}
\tilde{G}_{k}: T Y_{k} \rightarrow \mathbb{R}^{m_{k}-\left(\rho_{k}+q\right)} \times \mathcal{L}_{k} \\
(x, p) \mapsto \tilde{G}_{k}(x, p)=\left(g_{k}(x), \tilde{F}_{k}(x, p)\right) .
\end{gathered}
$$

Proposition 4.5. In an neighborhood of $\left(x_{0}, p_{0}\right)$ the $q-\pi$-submanifold $M_{k}$ is the zero set of the mapping $\tilde{G}_{k}$ and the $q-\pi$-submanifold $M_{k+1}$ is the zero set of the mapping $\tilde{F}_{k \mid T W_{k}}$.

Proof. According to the construction, the mapping $\tilde{G}_{k}$ is a submersion in a neighborhood of $\left(x_{0}, p_{0}\right)$, therefore the zero set of $\tilde{G}_{k}$ is a submanifold with the same dimension of $M$. Then we have only to prove that $M_{k} \subset \tilde{G}_{k}^{-1}(0)$ in a neighborhood of $\left(x_{0}, p_{0}\right)$ to set the equality $M_{k}=\tilde{G}_{k}^{-1}(0)$. For any point $(x, p)$ of $M_{k}$ in a neighborhood of $\left(x_{0}, p_{0}\right), G_{k}(x, p)=0$, therefore $(x, p, 0)$ belongs to $\tilde{M}_{k}$ which implies that $\tilde{F}_{k}(x, p)=0$. Lastly, $G_{k}(x, p)=0$ implies that $g_{k}(x)=0$. Then the inclusion has been proved. The reducibility assumption of $M_{k}$ in a neighborhood of $\left(x_{0}, p_{0}\right)$ gives

$$
\operatorname{rank}\left(\begin{array}{cc}
D^{2} g_{k}\left(x_{0}\right)\left(p_{0}, \bullet\right) & D g_{k}\left(x_{0}\right) \\
D_{x} \tilde{G}_{k}\left(x_{0}, p_{0}\right) & D_{p} \tilde{G}_{k}\left(x_{0}, p_{0}\right)
\end{array}\right)=2 m_{k}-\left(r_{k}+q\right) .
$$

Therefore, for any $\delta v$ of $\mathcal{L}_{k}$ there exists $(\delta x, \delta p)$ such that

$$
\begin{aligned}
D^{2} g_{k}\left(x_{0}\right)\left(p_{0}, \delta x\right)+D g_{k}\left(x_{0}\right) \delta p & =0 \\
D g_{k}\left(x_{0}\right) \delta p & =0 \\
D_{x} \tilde{F}_{k}\left(x_{0}, p_{0}\right) \delta x+D_{p} \tilde{F}_{k}\left(x_{0}, p_{0}\right) \delta p & =\delta v .
\end{aligned}
$$

In other words for any $\delta v$ of $\mathcal{L}_{k}$ there exists $(\delta x, \delta p)$ belonging to $T_{\left(x_{0}, p_{0}\right)} T W_{k}$ such that

$$
D_{x} \tilde{F}_{k}\left(x_{0}, p_{0}\right) \delta x+D_{p} \tilde{F}_{k}\left(x_{0}, p_{0}\right) \delta p=\delta v .
$$

Therefore the mapping $F_{k \mid T W_{k}}$ is a submersion in a neighborhood of $\left(x_{0}, p_{0}\right)$ and the zero set is $T W_{k} \cap V_{k}$.

We now see that, with a chart of $W_{k}$, we may define $G_{k+1}=\tilde{F}_{k \mid T W_{k}}$. ESAIM: COCV, MARCH 1998, VOL. 3, 49-81 


\section{APPENDIX}

\subsection{Proof OF MAIN RESULTS}

Proof of Theorem 2.4. Let $x(\cdot)$ be an admissible trajectory of $\mathcal{P}_{0}$ ( supposed continuously differentiable in the interval $[0,1]$ for reasons of convenience $\left.^{5}\right)$. Let $t_{0}$ belongs to the open interval $] 0,1\left[,\left(x_{0}, p_{0}\right)=\left(x\left(t_{0}\right), \dot{x}\left(t_{0}\right)\right)\right.$ and $W_{0}=\pi\left(V_{0}\right)$ be a local projection of $M_{0}$ at $\left(x_{0}, p_{0}\right)$, then for any $t$ in a open neighborhood of $t_{0},(x(t), \dot{x}(t))$ belongs to $V_{0}$. Consequently, $x(t)$ belongs to $W_{0}$ for any $t$ in an open neighborhood of $t_{0}$. Thus $(x(t), \dot{x}(t))$ belongs to $T W_{0} \cap V_{0}$ for any $t$ in an open neighborhood of $t_{0}$. Since $T W_{0} \cap V_{0}$ is equal to $M_{1}$ in an open neighborhood of $\left(x_{0}, p_{0}\right),(x(t), \dot{x}(t))$ belongs to $M_{1}$ for any $t$ in open neighborhood of $t_{0}$; this is the case for $t_{0}$. We have shown that for any $t_{0}$ belonging to the open interval $] 0,1\left[,\left(x\left(t_{0}\right), \dot{x}\left(t_{0}\right)\right)\right.$ belongs to $M_{1}$. Let us prove that $(x(0), \dot{x}(0))$ (resp. $\left.(x(1), \dot{x}(1))\right)$ belongs to $M_{1}$. Let $\left\{t_{n}\right\}_{n \geq 0}$ be a sequence of $] 0,1[$ converging to 0 (resp. 1 ), we then have

$$
\begin{gathered}
\lim _{n \rightarrow \infty}\left(x\left(t_{n}\right), \dot{x}\left(t_{n}\right)\right)=(x(0), \dot{x}(0)) \\
\text { (resp. } \left.\lim _{n \rightarrow \infty}\left(x\left(t_{n}\right), \dot{x}\left(t_{n}\right)\right)=(x(1), \dot{x}(1))\right) .
\end{gathered}
$$

But for any $n,\left(x\left(t_{n}\right), \dot{x}\left(t_{n}\right)\right)$ belongs to $M_{1}$ which is a closed subset of $M_{0}$, therefore $(x(0), \dot{x}(0))$ (resp. $(x(1), \dot{x}(1)))$ belongs to $M_{1}$. Thus we have shown that $x(\cdot)$ is an admissible trajectory of the problem $\mathcal{P}_{1}$. By induction we show that $x(\cdot)$ is an admissible trajectory of the problem $\mathcal{P}_{k}$ for any $k$. From the definition of the core $C(M), x(\cdot)$ is an admissible trajectory of $\mathcal{P}_{c}$. Let $\bar{x}(\cdot)$ be a strong (resp. weak) trajectory of $\mathcal{P}_{c}$, clearly it is an admissible trajectory of $\mathcal{P}_{0}$. Assume that $\bar{x}(\cdot)$ is not an strong (resp. weak) minimum of $\mathcal{P}_{0}$, then there exists an admissible trajectory $\tilde{x}(\cdot)$ of $\mathcal{P}_{0}$ such that $J(\tilde{x}(\cdot))<J(\bar{x}(\cdot))$, but any admissible trajectory of $\mathcal{P}_{0}$ is an admissible trajectory of $\mathcal{P}_{c}$. Therefore, $\tilde{x}(\cdot)$ is an admissible trajectory of $\mathcal{P}_{c}$ such that $J(\tilde{x}(\cdot))<J(\bar{x}(\cdot))$; then, we obtain a contradiction with the optimal character of $\bar{x}(\cdot)$.

Proof of Theorem 2.5. According to the proof of the theorem 3.10 the continuous mapping (resp. piecewise continuous) $u(\cdot)=H(x(\cdot), \dot{x}(\cdot)$ ) is the solution. The converse is direct.

REMARK 5.1. According to theorem 3.31 if we take another local projection $W^{\prime}=\pi\left(V^{\prime}\right)$ of $C(M)$ at $\left(x_{0}, p_{0}\right)$, another open set $O^{\prime}$ and another controlled vector field $\chi^{\prime}$ then the bundle isomorphism $h$ gives a bijection between the trajectories of $\chi$ and $\chi^{\prime}$. Then, the theorem 2.5 is independent of the choice of the triplets $(W, O, \chi)$.

\footnotetext{
${ }^{5}$ In the case of continuous and piecewise differentiable admissible trajectory $x(\cdot)$ we proceed from the same way for any interval $] 0, \tau_{1}[,] \tau_{k}, \tau_{k+1}[,] \tau_{n}, 1\left[\right.$, where $0 \leq \tau_{1}<\cdots<$ $\tau_{n} \leq 1$ are the points of discontinuity of $\dot{x}$ and for any admissible trajectory $x(\cdot)$ which is absolutely continuous we use the fact that there exists a denumerable sequence $\left(I_{n}\right)_{n \in N}$ of disjoint interval in $[0,1]$ such that the Lebesgue's measure of the set $I-\cup_{n} I_{n}$ is zero and the restriction of $(x(\cdot), \dot{x}(\cdot))$ in each interval $I_{n}$ is extended on the interval $\bar{I}_{n}$ by a continuous mapping $\left(x_{n}(\cdot), \dot{x}_{n}(\cdot)\right)$.
} 
Proof of Theorem 2.6. If $\bar{x}_{\mid I_{\varepsilon}}(\cdot)$ is not a strong minimum of the implicit Lagrange problem $\mathcal{P}_{c, \varepsilon}$ then there exists an admissible trajectory $\tilde{x}(\cdot)$ of $\mathcal{P}_{c, \varepsilon}$ such that

$$
\int_{\tau-\varepsilon}^{\tau+\varepsilon} L(\tilde{x}(t), \dot{\tilde{x}}(t)) d t<\int_{\tau-\varepsilon}^{\tau+\varepsilon} L(\bar{x}(t), \dot{\bar{x}}(t)) d t .
$$

Then, the construction of the admissible trajectory of the implicit Lagrange problem $\mathcal{P}_{c}$

$$
x^{\star}(t)= \begin{cases}\bar{x}(t) & \text { if } t \in[0, \tau-\varepsilon] \cup] \tau+\varepsilon, 1], \\ \tilde{x}(t) & \text { if } t \in] \tau-\varepsilon, \tau+\varepsilon],\end{cases}
$$

gives the inequality

$$
J\left(x^{\star}(\cdot)\right)<J(\bar{x}(\cdot))
$$

which contradicts the optimality of $\bar{x}(\cdot)$.

Proof of Theorem 2.7. Let $\bar{x}(\cdot)$ be a strong minimum of $\mathcal{P}_{c, \varepsilon}$ and $\bar{u}(\cdot)$ the corresponding control. Assume that the control $\bar{u}(\cdot)$ is not an optimal control of $\mathcal{P}_{e}$ then there exists an admissible control $\tilde{u}(\cdot)$ of the explicit optimal control problem $\mathcal{P}_{e}$ such that for the process $(\tilde{x}(\cdot), \tilde{u}(\cdot))$ we have the inequality

$$
\int_{\tau-\varepsilon}^{\tau+\varepsilon} L(\chi(\tilde{x}(t), \tilde{u}(t))) d t<\int_{\tau-\varepsilon}^{\tau+\varepsilon} L(\chi(\bar{x}(t), \bar{u}(t))) d t .
$$

According to the theorem 2.5 the trajectory $\tilde{x}(\cdot)$ is an admissible trajectory of $\mathcal{P}_{c, \varepsilon}$ such that

$$
\begin{aligned}
\int_{\tau-\varepsilon}^{\tau+\varepsilon} L(\tilde{x}(t), \dot{\tilde{x}}(t)) d t & =\int_{\tau-\varepsilon}^{\tau+\varepsilon} L(\chi(\tilde{x}(t), \tilde{u}(t))) d t \\
& <\int_{\tau-\varepsilon}^{\tau+\varepsilon} L(\chi(\bar{x}(t), \bar{u}(t))) d t \\
& =\int_{\tau-\varepsilon}^{\tau+\varepsilon} L(\bar{x}(t), \dot{\bar{x}}(t)) d t
\end{aligned}
$$

Which is impossible. Conversely, given $(\bar{x}(\cdot), \bar{u}(\cdot))$ an optimal process of the explicit optimal control problem $\mathcal{P}_{e}$, then according to theorem 2.5 the trajectory $\bar{x}(\cdot)$ is an admissible trajectory of $\mathcal{P}_{c, \varepsilon}$. If it is not a strong minimum then there exists an admissible trajectory $\tilde{x}(\cdot)$ of $\mathcal{P}_{c, \varepsilon}$ such that

$$
\int_{\tau-\varepsilon}^{\tau+\varepsilon} L(\tilde{x}(t), \dot{\tilde{x}}(t)) d t<\int_{\tau-\varepsilon}^{\tau+\varepsilon} L(\bar{x}(t), \dot{\bar{x}}(t)) d t .
$$

But, according to the theorem 2.5 for the trajectory $\tilde{x}(\cdot)$ there exists a unique control $\tilde{u}(\cdot)$ such that $(\tilde{x}(t), \dot{\tilde{x}}(t))=\chi(\tilde{x}(t), \tilde{u}(t))$. Thus, $(\tilde{x}(\cdot), \tilde{u}(\cdot))$ is an admissible process of $\mathcal{P}_{e}$ such that

$$
\begin{aligned}
\int_{\tau-\varepsilon}^{\tau+\varepsilon} L(\chi(\tilde{x}(t), \tilde{u}(t))) d t & =\int_{\tau-\varepsilon}^{\tau+\varepsilon} L(\tilde{x}(t), \dot{\tilde{x}}(t)) d t \\
& <\int_{\tau-\varepsilon}^{\tau+\varepsilon} L(\bar{x}(t), \dot{\bar{x}}(t)) d t \\
& =\int_{\tau-\varepsilon}^{\tau+\varepsilon} L(\chi(\bar{x}(t), \bar{u}(t))) d t
\end{aligned}
$$

which is impossible. 


\subsection{Pontryagin Maximum Principle}

For the classic problem of optimal control $([1,2,12,14])$ we are given a state variable $x$ in $\mathbb{R}^{n}$, a control variable $u$ belonging to a closed subset $U$ of $\mathbb{R}^{q}$, a vector field $f(x, u)$ of the state depending on the control variable, a startsubmanifold $X_{0}$ of $\mathbb{R}^{n}$, an endsubmanifold $X_{1}$ of $\mathbb{R}^{n}$ and a cost function $L(x, u)$. For any control $u$ belonging to $K C\left([0,1], \mathbb{R}^{q}\right)$ the set of piecewise continuous functions ${ }^{6}$ (resp. measurable and bounded ${ }^{7}$ ) the Cauchy's problem

$$
\left\{\begin{array}{l}
\dot{x}(t)=f(x(t), u(t)) \forall t \in[0,1] \text { (resp. a.e. on }[0,1]) \\
x(0)=a
\end{array}\right.
$$

admits an unique trajectory belonging to $K C^{1}\left([0,1], \mathbb{R}^{n}\right)$, the set of continuous and piecewise differentiable functions ${ }^{8}$ (resp. $A C\left([0,1], \mathbb{R}^{n}\right)$, the set of absolutely continuous functions $\left.{ }^{9}\right)$. The pairs trajectory/control $(x(\cdot), u(\cdot))$ are called the processes. A process is admissible if $u(t) \in U$ for any $t, x(0)$ belongs to $X_{0}$ and $x(1)$ belongs to $X_{1}$. For any admissible process $(x, u)$ we associate the cost

$$
J(x, u)=\int_{0}^{1} L(x(t), u(t)) d t .
$$

An admissible process $\bar{u}$ is optimal if $J(\bar{x}, \bar{u})$ is the minimum of $J$ on the set of admissible processes, namely the solution of the following problem

$$
\begin{aligned}
& \dot{x}(\cdot)=f(x(\cdot), u(\cdot)) \\
& x(0) \in X_{0} \\
& x(1) \in X_{1}
\end{aligned}
$$

The Pontryagin Maximum Principle gives the necessary conditions for optimality.

Theorem 5.2. (Maximum Principle) If $(\bar{x}(\cdot), \bar{u}(\cdot))$ is an optimal process for the problem $\mathcal{P}$, then there exists a non zero Lagrange multiplier

$$
\left(\bar{\psi}_{0}(\cdot), \bar{\psi}_{1}(\cdot), \cdots, \bar{\psi}_{n}(\cdot)\right)=\left(\psi_{0}(\cdot), \psi(\cdot)\right)
$$

belonging to $K C^{1}\left([0,1], \mathbb{R}^{n+1^{\star}}\right)$ (resp. $A C\left([0,1], \mathbb{R}^{n+1^{\star}}\right)$ ) and satisfying the following conditions

(a): for any $t$ belonging to $[0,1]$ (resp. a.e. on $[0,1]$ )

$$
\dot{\bar{\psi}}_{0}(t)=0, \dot{\bar{\psi}}_{i}(t)=-\frac{\partial H}{\partial x^{i}}\left(\bar{x}(t), \bar{u}(t), \bar{\psi}_{0}(t), \bar{\psi}(t)\right), i=1, \cdots, n .
$$

(b): for any $t$ belonging to $[0,1]$ (resp. a.e. on $[0,1]$ )

$$
H\left(\bar{x}(t), \bar{u}(t), \bar{\psi}_{0}(t), \bar{\psi}(t)\right)=\max _{u \in U} H\left(\bar{x}(t), u, \bar{\psi}_{0}(t), \bar{\psi}(t)\right) .
$$

(c): $\bar{\psi}_{0}(0) \leq 0, \bar{\psi}(0) \perp T_{\bar{x}(0)} X_{0}$ and $\bar{\psi}(1) \perp T_{\bar{x}(1)} X_{1}$

\footnotetext{
${ }^{6}$ For such control $u$, there exists a finite number of points $0<\tau_{1}<\cdots<\tau_{n}<1$ such that $u$ is continuous on any open interval $] 0, \tau_{1}[,] \tau_{k}, \tau_{k+1}[,] \tau_{n}, 1[$, and such that the right and left limits of $u$ at $\tau_{k}$ exist. We denote the set of points of continuity by $T$.

${ }^{7}$ For this class of control, $T$ is the set of Lebesgue points.

${ }^{8}$ For such trajectories $x$ the function $\dot{x}(\cdot)$ belongs to $K C\left([0,1], \mathbb{R}^{n}\right)$.

${ }^{9}$ Recall that for any $\varepsilon>0$ there exist a $\delta>0$ such that for any finite collection (]$a_{k}, b_{k}[)_{k=1, \cdots, n}$ of non overlapping open interval such that $\sum_{k=1}^{n}\left|b_{k}-a_{k}\right|<\delta$ then $\sum_{k=1}^{n}\left\|x\left(b_{k}\right)-x\left(a_{k}\right)\right\|<\varepsilon$.
} 
where $H\left(x, u, \psi_{0}, \psi\right)=\sum_{i=1}^{n} \psi_{i} f^{i}(x, u)+\psi_{0} L(x, u)$.

Proof of Theorem 2.7. See $[1,2,7,14]$ and $[8,13]$ for the nonsmooth case.

REMARK 5.3. a) As usual, we can only consider the two cases $\bar{\psi}_{0}(0)=0$ and $\bar{\psi}_{0}(0)=-1$ and then consider the following pseudo-Hamiltonian

$$
\begin{gathered}
H^{\psi_{0}}: T^{\star} \mathbb{R}^{n} \times U \rightarrow \mathbb{R} \\
H^{\psi_{0}}(x, \psi, u)=\sum_{i=1}^{n} \psi_{i} f^{i}(x, u)+\psi_{0} L(x, u)
\end{gathered}
$$

where $\psi_{0}=0,1$. The necessary conditions $(a)$ and $(b)$ become

(a'): the triplet $(\bar{x}(\cdot), \bar{\psi}(\cdot), \bar{u}(\cdot))$ is a trajectory of the controlled vector field

$$
\begin{gathered}
\overrightarrow{H^{\psi_{0}}}: T^{\star} \mathbb{R}^{n} \times U \rightarrow T\left(T^{\star} \mathbb{R}^{n}\right) \\
(x, \psi, u) \mapsto \overrightarrow{H^{\psi_{0}}}(x, \psi, u)=\sum_{i=1}^{n} \frac{\partial H^{\psi_{0}}}{\partial \psi_{i}}(x, \psi, u) \frac{\partial}{\partial x^{i}}-\sum_{i=1}^{n} \frac{\partial H^{\psi_{0}}}{\partial x^{i}}(x, \psi, u) \frac{\partial}{\partial \psi_{i}}
\end{gathered}
$$

where $\psi_{0}=0,1$.

$\left(b^{\prime}\right)$ : for any $t$ belonging to $[0,1]$ (resp. a.e. on $[0,1]$ )

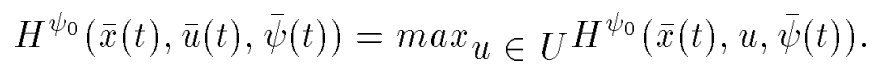

b) the trajectories $(\bar{x}(\cdot), \bar{\psi}(\cdot))$ which are the projection of a triplet

$$
(\bar{x}(\cdot), \bar{\psi}(\cdot), \bar{u}(\cdot))
$$

satisfying the conditions $\left(a^{\prime}\right),\left(b^{\prime}\right)$ and $(c)$ are called the extremals of $\mathcal{P}$.

I would like to express my sincere gratitude to Marc Chaperon and Pierre Rouchon for helpful discussions and encouragements.

\section{ReFERENCES}

[1] V. Alexeev, V.E. Galeev, V. Tikhomirov: Recueil de problèmes d'optimisation, Mir, Moscou, 1987.

[2] V. Alexeev, V. Tikhomirov, S. Fomin: Optimal control, Plenum Publishing Corporation, New York, 1987.

[3] G.A. Bliss: The problem of Lagrange in the calculus of variations, American Journal of Mathematics 52 (1930), 673-744.

[4] G.A. Bliss: Lectures on the calculus of variations, University of Chicago press, Chicago, 1946.

[5] C. Carathéodory: Calculus of variations and partial differential equations of the first order, Series in Mathematical Physics, Holden-Day, San francisco, Cambridge, London, Amsterdam, 1967.

[6] E. Cartan: Sur l'intégration de certains systèmes indéterminés d'équations différentielles, vol. 2, CNRS, Paris, 1984.

[7] L. Cesari: Optimization-theory and applications, Applications of Mathematics, Springer-Verlag, New York Heidelberg Berlin, 1983.

[8] F. Clarke: Optimization and nonsmooth analysis, Les publications du Centre de Recherche Mathématiques de l'Université de Montréal, 1989.

[9] J. Dieudonné: Eléments d'analyse, vol. 3, Gauthier-Villars, Paris, 1970.

[10] P.A. Griffiths: Exterior differential systems and the calculus of variations, Birkäuser, Boston, 1983.

[11] J. Hadamard: Leçons sur le calcul des variations, Herman, Paris, 1940.

ESAIM: COCV, MARCH 1998, VOL. 3, 49-81 
[12] V. Jurdjevic: Geometric control theory, Cambridge Studies in Advanced Mathematics, Cambridge University Press, Cambridge, 1997.

[13] Ph. Loewen: Optimal control via nonsmooth analysis, CRM Proceedings and Lecture Notes, vol. 2, American Mathematical Society, Providence, Rhode Island USA, 1993.

[14] L. Pontryagin et al.: Théorie mathématique de processus optimaux, Mir, Moscou, 1974.

[15] P.-J. Rabier, W.C. Rheinboldt: A general existence and uniqueness theory for implicit differential-algebraic equations, Differential Integral Equations 4 (1991), 563582 .

[16] P.-J. Rabier , W.C. Rheinboldt: A geometric treatment of implicit differentialalgebraic equations, Journal of Differential Equations 109 (1994), 110-146. 